\title{
IMBEDDING THEOREMS FOR SOBOLEV SPACES ON DOMAINS WITH PEAK AND ON HÖLDER DOMAINS
}

\author{
V. G. MAZ'YA AND S. V. POBORCHI
}

\begin{abstract}
Necessary and sufficient conditions are obtained for the continuity and compactness of the imbedding operators $W_{p}^{l}(\Omega) \rightarrow L_{q}(\Omega)$ and $W_{p}^{l}(\Omega) \rightarrow C(\Omega) \cap$ $L_{\infty}(\Omega)$ for a domain with an outward peak. More simple sufficient conditions are presented. Applications to the solvability of the Neumann problem for elliptic equations of order $2 l, l \geq 1$, for a domain with peak are given. An imbedding theorem for Sobolev spaces on Hölder domains is stated.
\end{abstract}

\section{§1. Introduction AND STATEMENT OF PRINCIPAL RESUlTS}

Sobolev's theorem on imbedding of the space $W_{p}^{l}(\Omega)$ in $L_{q}(\Omega)$ was proved by Sobolev [1, 2] and supplemented by Gagliardo [3] in the case where the domain $\Omega \subset \mathbb{R}^{n}$ has the cone property. If $l$ is a positive integer, $1 \leq p<\infty$, and $l p<n$, then the exponent $q$ in the above imbedding takes the maximal possible value $q=n p /(n-l p)$.

In [12, Maz'ya obtained a necessary and sufficient condition for the continuity of the imbedding operator $W_{p}^{1}(\Omega) \rightarrow L_{q}(\Omega)$ in the case of a general domain $\Omega \subset \mathbb{R}^{n}$. These conditions are either isoperimetric (for $p=1$ ), or capacitary (for $p>1$ ) inequalities. As an application of these results, a maximal exponent $q$ was found for which $W_{p}^{1}(\Omega)$ is continuously imbedded in $L_{q}(\Omega)$ for the power cusp domain

$$
\Omega=\left\{x=\left(x^{\prime}, x_{n}\right) \in \mathbb{R}^{n}: x_{n} \in(0,1),\left|x^{\prime}\right|<\varphi\left(x_{n}\right)\right\}, \quad n \geq 2,
$$

where $\varphi(t)=$ const $\cdot t^{\lambda}, \lambda>1$. This maximal $q$ looks like this:

$$
q=(1+\lambda(n-1)) p /(1+\lambda(n-1)-p) \quad \text { for } 1 \leq p<1+\lambda(n-1) .
$$

Later on, the imbedding theorem $W_{p}^{l}(\Omega) \subset L_{q}(\Omega)$ with Sobolev's limit exponent was extended beyond the class of domains with the cone property, specifically, to domains with the flexible cone property (Besov 4), and to John domains (Reshetnyak 6], Bojarski [7]). Moreover, it turned out (Buckley and Koskela [8]) that, in a sense, the John domains form the largest class of domains supporting the imbedding theorem with Sobolev's limit exponent.

Hajłasz and Koskela [9] established the continuous imbedding $W_{p}^{1}(\Omega) \subset L_{q}(\Omega)$ for $\lambda$ John domains with $\lambda>1$. In that paper, $q$ is maximal for $p=1$ and "almost maximal" for $p>1$. The maximal possible $q$ for the above imbedding with $p>1$ was obtained by Kilpeläinen and Malý [10]. Besov [5] proved the continuous imbedding theorem $W_{p}^{l}(\Omega) \subset$ $L_{q}(\Omega)$ for a certain class of domains containing all $\lambda$-John domains. In particular, it was shown [5] that for the $\lambda$-John domains with $\lambda>1$ and $l p<\lambda(n-1)+1$ the space $W_{p}^{l}(\Omega)$ is continuously imbedded in $L_{q}(\Omega)$ for $q=n p /(1+\lambda(n-1)-l p)$. This exponent is maximal in general (see Poborchiı [11], Labutin [22]).

2000 Mathematics Subject Classification. Primary 46E35.

Key words and phrases. Sobolev spaces, imbedding theorems, irregular boundary, domain with peak. 
We observe that the domain (1.1) is $\lambda$-John, but for it the limit exponent $q$ with $W_{p}^{l}(\Omega) \subset L_{q}(\Omega)$ is greater than that for the class of $\lambda$-John domains. The domains with outward peaks provide the simplest examples where Sobolev's theorem fails. A great deal of attention has been paid to conditions ensuring the continuity of the imbedding operators $W_{p}^{l}(\Omega) \rightarrow L_{q}(\Omega)$ (or $\left.W_{p}^{l}(\Omega) \rightarrow C(\Omega) \cap L_{\infty}(\Omega)\right)$ for such domains. Labutin [20] proved the imbedding theorem with the maximal exponent for a class of domains which may have outward power peaks. Concerning similar results for power cusps, see also Adams [23, Subsections 5.35, 5.36], Globenko [19], Fukushima and Tomisaki [24]. In this connection, we mention the paper [21] by Labutin, where the imbedding of the Sobolev space in $L_{q}$ was studied for Hölder domains.

As a consequence of a general capacitary criteria, in [13, 14] and [15, 4.4, 5.1] Maz'ya obtained (among other things) necessary and sufficient conditions for the continuity of the imbeddings of $W_{p}^{1}(\Omega)$ in $L_{q}(\Omega)$ and in $C(\Omega) \cap L_{\infty}(\Omega)$ for the domain (1.1). In the case of a continuous positive convex function $\varphi, \varphi(0)=0$, these conditions have the form

$$
\sup _{z \in(0,1)}\left\{\left(\int_{0}^{z} \varphi(t)^{n-1} d t\right)^{\frac{1}{q}}\left(\int_{z}^{1} \varphi(t)^{\frac{n-1}{1-p}} d t\right)^{\frac{p-1}{p}}\right\}<\infty
$$

for the continuity of the imbedding operator $W_{p}^{1}(\Omega) \rightarrow L_{q}(\Omega), 1<p \leq q<\infty$, and

$$
\int_{0}^{1} \varphi(t)^{\frac{n-1}{1-p}} d t<\infty
$$

for the continuity of the imbedding operator $W_{p}^{1}(\Omega) \rightarrow C(\Omega) \cap L_{\infty}(\Omega)$.

In the authors' paper [17. (see also [18, Subsections 8.2, 8.3]) this result was extended to the spaces $W_{p}^{l}(\Omega), l \geq 1$, and to domains $\Omega$ with the vertex of an outward peak described by some function $\varphi$. However, for $l>1$ the additional condition $c^{-1} \leq \varphi(2 t) / \varphi(t) \leq c, c=$ const $>0$, was imposed on $\varphi$. In the present paper we lift this restriction.

The precise statement will be given below. Now we introduce some notation.

Let $x=\left(x_{1}, \ldots, x_{n}\right)$ be a point in the $n$-dimensional Euclidean space $\mathbb{R}^{n}$ with the norm $|x|=\left(x_{1}^{2}+\cdots+x_{n}^{2}\right)^{1 / 2}$. For $r>0$, we denote by $B_{r}(x)$ the open ball in $\mathbb{R}^{n}$ with center $x$ and radius $r$. In what follows, $B_{r}=B_{r}(0)$. We write $B_{r}^{(n)}$ to emphasize the dimension of the ball.

Let $\mathbb{Z}_{+}^{n}$ denote the subspace of $\mathbb{R}^{n}$ formed by the vectors with nonnegative integral components. An element $\alpha=\left(\alpha_{1}, \ldots, \alpha_{n}\right) \in \mathbb{Z}_{+}^{n}$ is called a multiindex, and the length of $\alpha$ is $|\alpha|=\alpha_{1}+\cdots+\alpha_{n}$. If $x \in \mathbb{R}^{n}$ and $\alpha \in \mathbb{Z}_{+}^{n}$, then

$$
\alpha !=\alpha_{1} ! \cdots \alpha_{n} !, \quad x^{\alpha}=x_{1}^{\alpha_{1}} \cdots x_{n}^{\alpha_{n}} .
$$

The partial derivatives will be written as

$$
D^{\alpha}=\frac{\partial^{|\alpha|}}{\partial x_{1}^{\alpha_{1}} \cdots \partial x_{n}^{\alpha_{n}}}, \quad \alpha \in \mathbb{Z}_{+}^{n} .
$$

If $l$ is a positive integer, the gradient of order $l$ of a function $u$ is $\nabla_{l} u=\left\{D^{\alpha} u\right\}_{|\alpha|=l}$. By definition, $\left|\nabla_{l} u\right|=\left(\sum_{|\alpha|=l}\left|D^{\alpha} u\right|^{2}\right)^{1 / 2}$.

By $\mathcal{P}_{l}^{(n)}$ (or simply by $\mathcal{P}_{l}$ for fixed $n$ ) we denote the class of polynomials on $\mathbb{R}^{n}$ of degree at most $l, l=0,1, \ldots$.

Let $\Omega$ be a domain in $\mathbb{R}^{n}$ (i.e., an open connected set) and $l \geq 0$ an integer. The symbol $C^{l}(\Omega)$ designates the class of (real-valued) functions $u \in C(\Omega)$ that have derivatives $D^{\alpha} u \in C(\Omega)$ for $|\alpha| \leq l$. Furthermore, the set of infinitely differentiable functions in $\Omega$ is denoted by $C^{\infty}(\Omega)$. Next, $C_{0}^{\infty}(\Omega)$ is the subset of $C^{\infty}(\Omega)$ formed by the functions compactly supported in $\Omega$. The space $C^{l}(\bar{\Omega})$ consists of the elements in $C^{l}(\Omega)$ whose 
derivatives of orders $0, \ldots, l$ admit a continuous extension to $\bar{\Omega}$. The space of restrictions of the functions in $C^{\infty}\left(\mathbb{R}^{n}\right)$ to $\Omega$ is denoted by $C^{\infty}(\bar{\Omega})$.

Let $1 \leq p \leq \infty$. The space $L_{p}(\Omega)$ consists of all Lebesgue measurable functions $u$ on $\Omega$ for which

$$
\|u\|_{L_{p}(\Omega)}=\left(\int_{\Omega}|u(x)|^{p} d x\right)^{1 / p}<\infty, \quad p<\infty
$$

and

$$
\|u\|_{L_{\infty}(\Omega)}=\operatorname{ess} \sup \{|u(x)|: x \in \Omega\}<\infty .
$$

For brevity, we also write $\|\cdot\|_{p, \Omega}$ instead of $\|\cdot\|_{L_{p}(\Omega)}$.

Let $L_{p, \text { loc }}(\Omega)$ be the class of all measurable functions on $\Omega$ that are integrable with exponent $p$ (essentially bounded for $p=\infty$ ) on every compact subset of $\Omega$.

Suppose that $\Omega$ is a domain in $\mathbb{R}^{n}, l=1,2, \ldots$, and $1 \leq p \leq \infty$. The space $L_{p}^{l}(\Omega)$ consists of the elements of $L_{p \text {, loc }}(\Omega)$ whose gradient of order $l$ belongs to $L_{p}(\Omega)$. By definition, $W_{p}^{l}(\Omega)=L_{p}(\Omega) \cap L_{p}^{l}(\Omega)$. The space $W_{p}^{l}(\Omega)$ is endowed with the norm

$$
\|u\|_{W_{p}^{l}(\Omega)}=\|u\|_{p, \Omega}+\left\|\nabla_{l} u\right\|_{p, \Omega},
$$

and $L_{p}^{l}(\Omega)$ with the norm

$$
\|u\|_{L_{p}^{l}(\Omega)}=\|u\|_{p, D}+\left\|\nabla_{l} u\right\|_{p, \Omega},
$$

where $D$ is an inner subdomain of $\Omega$, i.e., $D$ is bounded and $\bar{D} \subset \Omega$. Different subdomains $D$ induce equivalent norms (see [15, Subsection 1.1.13]). The conventional notation $L_{p}^{0}(\Omega)=W_{p}^{0}(\Omega)=L_{p}(\Omega)$ is used if $l=0$.

Now we describe some classes of domains. A domain $\Omega \subset \mathbb{R}^{n}$ is said to have the cone property if every point in $\Omega$ is the vertex of a closed cone contained in $\Omega$ and congruent to the cone

$$
\left\{x \in \mathbb{R}^{n}: x_{1}^{2}+\cdots+x_{n-1}^{2} \leq a x_{n}^{2}, 0 \leq x_{n} \leq b\right\}, \quad a, b=\text { const }>0 .
$$

A bounded domain $\Omega \subset \mathbb{R}^{n}$ belongs to the class $C^{0,1}$ if every point of $\partial \Omega$ has a neighborhood $U$ such that the set $U \cap \Omega$ can be represented by the inequality $x_{n}<$ $f\left(x_{1}, \ldots, x_{n-1}\right)$ for some local Cartesian system, where $f$ is a function defined on a domain $G \subset \mathbb{R}^{n-1}$ and satisfying the Lipschitz condition on $G$.

We introduce the class of domains of the form

$$
\Omega=\left\{x=(y, z) \in \mathbb{R}^{n}: z \in(0,1), y / \varphi(z) \in \omega\right\}, \quad n \geq 2,
$$

assuming that $\omega \subset \mathbb{R}^{n-1}$ is a bounded domain with the cone property and $\varphi$ is a monotone increasing function on $[0,1]$ satisfying the Lipschitz condition and such that

$$
\varphi(0)=\lim _{z \rightarrow+0} \varphi^{\prime}(z)=0 .
$$

We say that $\Omega$ is a peak in $\mathbb{R}^{n}$. For simplicity of presentation, in what follows it is assumed that $\bar{\omega} \subset B_{1}^{(n-1)}$.

In $\S 5$ below, yet another class of domains with the so-called Hölder boundaries will be described.

Now we state the principal results of the paper that pertain to the peak (1.3).

Theorem 1.1. Suppose $\Omega$ is a peak in $\mathbb{R}^{n}, l=1,2, \ldots$, and $1<p \leq q<\infty$. The space $W_{p}^{l}(\Omega)$ is continuously imbedded in $L_{q}(\Omega)$ if and only if $A_{0}, A_{1}<\infty$, where

$$
\begin{aligned}
A_{\gamma} & =\sup _{z \in(0,1)} A_{\gamma}(z), \\
A_{\gamma}(z) & =\left(\int_{0}^{z}(z-t)^{q(l-1)(1-\gamma)} \varphi(t)^{n-1} d t\right)^{\frac{1}{q}}\left(\int_{z}^{1} \varphi(t)^{\frac{n-1}{1-p}}(t-z)^{\frac{p(l-1) \gamma}{p-1}} d t\right)^{\frac{p-1}{p}} .
\end{aligned}
$$


The imbedding operator $W_{1}^{l}(\Omega) \rightarrow L_{q}(\Omega), 1 \leq q<\infty$, is continuous if and only if

$$
\sup _{z \in(0,1)} \varphi(z)^{1-n}\left(\int_{0}^{z}(z-t)^{(l-1) q} \varphi(t)^{n-1} d t\right)^{1 / q}<\infty .
$$

The compactness criterion can be formulated as follows.

Theorem 1.2. Suppose $1<p \leq q<\infty$ and $l=1,2, \ldots$ The imbedding operator $W_{p}^{l}(\Omega) \rightarrow L_{q}(\Omega)$ for an $n$-dimensional peak $\Omega$ is compact if and only if

$$
\lim _{z \rightarrow+0} A_{0}(z)=\lim _{z \rightarrow+0} A_{1}(z)=0
$$

where $A_{\gamma}(z)$ is the same as in the preceding theorem. In the case where $1 \leq q<\infty$, the imbedding operator $W_{1}^{l}(\Omega) \rightarrow L_{q}(\Omega)$ is compact if and only if

$$
\lim _{z \rightarrow+0} \varphi(z)^{1-n}\left(\int_{0}^{z}(z-t)^{(l-1) q} \varphi(t)^{n-1} d t\right)^{1 / q}=0 .
$$

The following assertion is a consequence of the two above.

Corollary 1.1. Let $\varphi$ be the function describing the cusp in (1.3), and let

$$
\Phi(z)=\varphi(z)^{(n-1)\left(q^{-1}-p^{-1}\right)} z^{l-p^{-1}+q^{-1}}
$$

for $1 \leq p \leq q<\infty$. Then the inequality

$$
\sup \{\Phi(z): z \in(0,1)\}<\infty
$$

ensures the continuity of the imbedding operator $W_{p}^{l}(\Omega) \rightarrow L_{q}(\Omega)$, and the relation

$$
\lim _{z \rightarrow+0} \Phi(z)=0
$$

ensures its compactness. Conditions (1.8), (1.9) are also necessary if $\varphi(2 z) \leq \mathrm{const} \cdot \varphi(z)$, $z \in(0,1 / 2)$.

The statement below gives necessary and sufficient conditions for the continuity and compactness of the imbedding operator $W_{p}^{l}(\Omega) \rightarrow C(\Omega) \cap L_{\infty}(\Omega)$ for a peak of the form (1.3).

Theorem 1.3. If $p \in(1, \infty)$ and $l=1,2, \ldots$, then the imbedding operator $W_{p}^{l}(\Omega) \rightarrow$ $C(\Omega) \cap L_{\infty}(\Omega)$ for an $n$-dimensional peak $\Omega$ is continuous if and only if

$$
\int_{0}^{1} \frac{z^{(l-1) p /(p-1)}}{\varphi(z)^{(n-1) /(p-1)}} d z<\infty .
$$

This imbedding operator is compact. The space $W_{1}^{l}(\Omega)$ is continuously imbedded in $C(\Omega) \cap$ $L_{\infty}(\Omega)$ if and only if

$$
\sup \left\{z^{l-1} \varphi(z)^{1-n}: z \in(0,1)\right\}<\infty
$$

and the condition

$$
\lim _{z \rightarrow+0} z^{l-1} \varphi(z)^{1-n}=0
$$

is necessary and sufficient for the last-mentioned imbedding to be compact. If the domain $\omega$ in (1.3) is of class $C^{0,1}$, then the imbedding operator $W_{p}^{l}(\Omega) \rightarrow C(\bar{\Omega})$ is continuous (compact) under the above assumptions of the theorem.

As an application of Theorems 1.1 and 1.2, we consider the following example. 
Example. Sobolev's theorem implies that, for a bounded domain $\Omega \subset \mathbb{R}^{n}$ with the cone property, $W_{p}^{l}(\Omega)$ is continuously imbedded in $L_{q}(\Omega)$ with $q=n p /(n-l p)$ if $l p<n$. This exponent $q$ is the maximal possible, and the imbedding operator $W_{p}^{l}(\Omega) \rightarrow L_{q}(\Omega)$ is not compact. Now we verify that if $\Omega$ fails to have the cone property, then the limit exponent $q$ for the imbedding $W_{p}^{l}(\Omega) \subset L_{q}(\Omega), l p<n$, can take any value in $(p, n p /(n-l p))$, and the imbedding with this limit exponent can be compact.

Indeed, suppose $p \geq 1, l p<n$, and $q \in(p, n p /(n-l p))$. Then there exists a bounded domain $\Omega \subset \mathbb{R}^{n}$ such that

1) the imbedding operator $W_{p}^{l}(\Omega) \rightarrow L_{q}(\Omega)$ is compact;

2 ) the space $W_{p}^{l}(\Omega)$ is not imbedded in $L_{r}(\Omega)$ for $r>q$.

By Theorems 1.1 and 1.2 (see also Corollary 1.1) as $\Omega$ we can take the domain (1.3) with

$$
\varphi(z)=z^{\lambda}(1-\log z), \quad \lambda=(l-1 / p+1 / q) /((n-1)(1 / p-1 / q)) .
$$

The next two sections are auxiliary. $\S 4$ contains the proof of Theorems 1.1-1.3. In $\S 4$ we also consider an application of Theorem 1.1 to the Neumann problem for elliptic equations of order $2 l, l \geq 1$. In $\S 5$ we state the imbedding theorems $W_{p}^{l}(\Omega) \subset L_{q}(\Omega)$ and $W_{p}^{l}(\Omega) \subset C(\bar{\Omega})$ for Hölder domains.

\section{§2. The Friedrichs inequality For a domain With PEAK AND SMOOTHING OF A FUNCTION THAT DESCRIBES THE PEAK}

The lemma stated below plays an important role in what follows.

Lemma 2.1. Let $\Omega$ be a peak in $\mathbb{R}^{n}$. Suppose that $u \in L_{p}^{l}(\Omega)$ and $u(y, z)=0$ in the vicinity of $z=1$. Then the estimate

$$
\|u\|_{p, \Omega} \leq c\left\|\nabla_{l} u\right\|_{p, \Omega}, \quad 1 \leq p \leq \infty, \quad l=1,2, \ldots,
$$

is true with a constant $c$ independent of $u$.

Proof. Since all intermediate derivatives $D^{\alpha} u$ exist and belong to $L_{p, \text { loc }}(\Omega),|\alpha|=0, \ldots$, $l-1$ (see, e.g., Maz'ya [15, Subsection 1.1.2]), it suffices to consider the case where $l=1$. Then the general case will follow easily by induction on $l$. We have

$$
u(\varphi(z) \eta, z)=-\int_{z}^{1} \frac{\partial}{\partial t}(u(\varphi(t) \eta, t)) d t
$$

for almost all $\eta \in \omega$ and almost all $z \in(0,1)$. Therefore, the required inequality is true for $p=\infty$. Let $p<\infty$. Then

$$
|u(\varphi(z) \eta, z)|^{p} \leq c \int_{z}^{1}|(\nabla u)(\varphi(t) \eta, t)|^{p} d t
$$

whence

$$
\begin{aligned}
\|u\|_{p, \Omega}^{p} & =\int_{0}^{1} \varphi(z)^{n-1} d z \int_{\omega}|u(\varphi(z) \eta, z)|^{p} d \eta \\
& \leq c \int_{0}^{1} \varphi(z)^{n-1} d z \int_{z}^{1} d t \int_{\omega}|(\nabla u)(\varphi(t) \eta, t)|^{p} d \eta .
\end{aligned}
$$

The right-hand side does not exceed

$$
c \int_{0}^{1} \varphi(t)^{n-1} d t \int_{\omega}|(\nabla u)(\varphi(t) \eta, t)|^{p} d \eta=c\|\nabla u\|_{p, \Omega}^{p},
$$

and the proof of the lemma is finished. 
The next assertion is a consequence of Lemma 2.1 and Sobolev's theorem on equivalent norms in $W_{p}^{l}(\Omega)$ (see [2, §9] and [15, Subsection 1.1.15]).

Corollary 2.1. For an $n$-dimensional peak $\Omega$, the spaces $L_{p}^{l}(\Omega), W_{p}^{l}(\Omega)$, and $\bigcap_{k=0}^{l} L_{p}^{k}(\Omega)$ coincide for all $l=1,2, \ldots$ and $1 \leq p \leq \infty$. In particular, the norm (1.2) is equivalent to the norm $\sum_{|\alpha| \leq l}\left\|D^{\alpha} u\right\|_{p, \Omega}$ and to any norm of the form $\|u\|_{p, G}+\left\|\nabla_{l} u\right\|_{p, \Omega}$, where $G$ is an inner subdomain of $\Omega$, i.e., $\bar{G} \subset \Omega$.

Let $\varphi$ be a function describing the peak in (1.3). The following lemma says that, in the proof of the imbedding theorem, $\varphi$ can be replaced by another function satisfying some additional conditions.

Lemma 2.2. Let $\varphi$ be a monotone increasing function on $[0,1]$ satisfying the Lipschitz condition and such that $\varphi(0)=\lim _{z \rightarrow+0} \varphi^{\prime}(z)=0$. Then for any positive integer $l$ there exists a monotone increasing function $f \in C^{l}((0,1])$ and a constant $c>0$ depending only on $\varphi$ and $l$ such that $\lim _{z \rightarrow+0} f(z)=\lim _{z \rightarrow+0} f^{\prime}(z)=0$, and

$$
\begin{gathered}
c^{-1} \varphi(z) \leq f(z) \leq c \varphi(z), \\
\left|f^{(k)}(z)\right| \leq c f(z)^{1-k}, \quad k=1, \ldots, l,
\end{gathered}
$$

for all $z \in(0,1]$.

Proof. Suppose $\psi \in C^{l}\left(\mathbb{R}^{1}\right), \psi(t)=0$ for $t \leq 0$, and $\psi(t)=1$ for $t \geq 1$. Let $\psi$ be monotone increasing on $[0,1]$. We construct a sequence $\left\{z_{i}\right\}$ by

$$
z_{0}=1, \quad z_{i+1}+\varphi\left(z_{i+1}\right)=z_{i}, \quad i=0,1, \ldots .
$$

Clearly, $\left\{z_{i}\right\}$ is monotone decreasing and $z_{i} \rightarrow 0$; furthermore,

$$
z_{i+1}^{-1} z_{i} \rightarrow 1, \quad \varphi\left(z_{i+1}\right)^{-1} \varphi\left(z_{i}\right) \rightarrow 1 .
$$

Putting

$$
\psi_{0}(z)=\psi\left(\left(z-z_{1}\right) /\left(z_{0}-z_{1}\right)\right), \quad z \in(0,1],
$$

we define $\psi_{i}$ for $i=1,2, \ldots$ by

$$
\psi_{i}(z)= \begin{cases}\psi\left(\left(z-z_{i+1}\right) /\left(z_{i}-z_{i+1}\right)\right) & \text { if } z \in\left(0, z_{i}\right], \\ 1-\psi\left(\left(z-z_{i}\right) /\left(z_{i-1}-z_{i}\right)\right) & \text { if } z \in\left(z_{i}, 1\right] .\end{cases}
$$

Then $0 \leq \psi_{i} \leq 1, \psi_{i} \in C^{l}((0,1])$ for $i \geq 0$, and also

$$
\operatorname{supp} \psi_{i}=\left[z_{i+1}, z_{i-1}\right] \text { for } i \geq 1, \quad \sum_{i=0}^{\infty} \psi_{i}(z)=1, z \in(0,1] .
$$

We claim that $f$ can be given by the formula

$$
f(z)=\sum_{i=0}^{\infty} \varphi\left(z_{i}\right) \psi_{i}(z), \quad z \in(0,1] .
$$

Obviously, $f \in C^{l}((0,1])$. Next, if $z \in\left[z_{i+1}, z_{i}\right], i \geq 0$, then $\psi_{i}(z)+\psi_{i+1}(z)=1$. Therefore,

$$
f(z)=\varphi\left(z_{i}\right) \psi_{i}(z)+\varphi\left(z_{i+1}\right) \psi_{i+1}(z) \in\left[\varphi\left(z_{i+1}\right), \varphi\left(z_{i}\right)\right]
$$

whence

$$
\varphi\left(z_{i+1}\right) / \varphi\left(z_{i}\right) \leq f(z) / \varphi(z) \leq \varphi\left(z_{i}\right) / \varphi\left(z_{i+1}\right), \quad z \in\left[z_{i+1}, z_{i}\right] .
$$

Thus, (2.1) is true with $c=\sup \left\{\varphi\left(z_{i}\right) / \varphi\left(z_{i+1}\right): i \geq 0\right\}$. In particular, $f(z) \rightarrow 0$ as $z \rightarrow+0$.

To check the monotonicity of $f$, we observe that

$$
f(z)=\varphi\left(z_{i+1}\right)+\left(\varphi\left(z_{i}\right)-\varphi\left(z_{i+1}\right)\right) \psi_{i}(z), \quad z \in\left[z_{i+1}, z_{i}\right], \quad i \geq 0 .
$$


Consequently, $f$ is monotone increasing, because so is $\psi_{i}$ on $\left[z_{i+1}, z_{i}\right]$. Furthermore, for $k=1, \ldots, l$ and $z \in\left[z_{i+1}, z_{i}\right]$ we have

$$
f^{(k)}(z)=\frac{\varphi\left(z_{i}\right)-\varphi\left(z_{i+1}\right)}{\left(z_{i}-z_{i+1}\right)^{k}} \psi^{(k)}\left(\frac{z-z_{i+1}}{z_{i}-z_{i+1}}\right) .
$$

Hence, for the same $z$ and $k$,

$$
\left|f^{(k)}(z)\right| \leq c \varphi\left(z_{i+1}\right)^{1-k} \operatorname{ess} \sup \left\{\varphi^{\prime}(t): t \in\left(z_{i+1}, z_{i}\right)\right\} .
$$

This implies that $\lim _{z \rightarrow+0} f^{\prime}(z)=0$ and (2.2) is fulfilled, completing the proof of the lemma.

\section{§3. Estimates For Derivatives of A FUnCtion AVERAGED WITH RESPECT TO PART OF THE VARIABLES}

Here we prove an auxiliary assertion to be used in the proof of Theorem 1.1. We start with a remark that follows from the theorem on equivalent norms in Sobolev spaces.

Remark 3.1. Let $D \subset \mathbb{R}^{n}$ be a bounded domain with the cone property. Suppose that $\Pi: W_{p}^{l}(D) \rightarrow \mathcal{P}_{l-1}$ is a continuous linear map satisfying $\Pi^{2}=\Pi$ (i.e., $\Pi$ is a projection of $W_{p}^{l}(\Omega)$ to $\left.\mathcal{P}_{l-1}\right)$. Then, for all $u \in W_{p}^{l}(D)$ and $k=0, \ldots, l-1$, we have the estimate

$$
\left\|\nabla_{k}(u-\Pi u)\right\|_{p, D} \leq c\left\|\nabla_{l} u\right\|_{p, D}, \quad k=0, \ldots, l-1,
$$

with a constant $c$ independent of $u$ (see [2, §9] and [15, Subsection1.1.15]). This estimate is known as the generalized Poincaré inequality.

Example. We construct an example of a projection $W_{p}^{l}(D) \rightarrow \mathcal{P}_{l-1}$ suitable for any domain $D$. Let $K \in C_{0}^{\infty}(D)$,

$$
\int K(x) d x=1 \text { and } \int K(x) x^{\alpha} d x=0 \text { for all } \alpha \in \mathbb{Z}_{+}^{n} \text { with } 1 \leq|\alpha| \leq l-1 .
$$

Then the required map $W_{p}^{l}(D) \ni u \mapsto \Pi u \in \mathcal{P}_{l-1}$ can be defined by

$$
(\Pi u)(x)=\sum_{|\alpha|<l} \frac{x^{\alpha}}{\alpha !} \int_{D}\left(D^{\alpha} u\right)(y) K(y) d y=\sum_{|\alpha|<l} \frac{(-x)^{\alpha}}{\alpha !} \int_{D} u(y) D^{\alpha} K(y) d y .
$$

Let $l$ be a positive integer and $f$ a monotone increasing function in $C^{l}(0,1)$ such that

$$
\lim _{z \rightarrow 0} f(z)=0, \quad\left|f^{(k)}(z)\right| \leq \operatorname{const} f(z)^{1-k}, \quad k=1, \ldots, l, \quad z \in(0,1) .
$$

Let $K \in C_{0}^{\infty}\left(B_{1}^{(n-1)}\right)$ satisfy

$$
\int K(y) y^{\alpha} d y=0, \quad \alpha \in \mathbb{Z}_{+}^{n-1}, \quad 1 \leq|\alpha| \leq l-1 .
$$

We consider the domain

$$
G=\left\{x=(y, z) \in \mathbb{R}^{n}: z \in(0,1),|y|<f(z)\right\}, \quad n \geq 2,
$$

and define

$$
(T v)(z)=\int_{|\eta|<1} K(\eta) v(f(z) \eta, z) d \eta, \quad z \in(0,1),
$$

for $v \in L_{p}(G)$. In what follows, $c$ designates various positive constants depending only on $n, l, p, K$, and $f$. 
Lemma 3.1. Let $u \in W_{p}^{l}(G), 1 \leq p \leq \infty$. Suppose $\alpha \in \mathbb{Z}_{+}^{n-1},|\alpha|<l$, and let $D^{\alpha} u$ denote the derivative of $u$ with respect to $y_{1}, y_{2}, \ldots, y_{n-1}$. Then the function

$$
(0,1) \ni z \mapsto u_{\alpha}(z)=\left(T\left(D^{\alpha} u\right)\right)(z)
$$

has all derivatives $u_{\alpha}^{(s)} \in L_{p, \mathrm{loc}}(0,1), s=0, \ldots, l$. Furthermore, if $l-|\alpha| \leq s \leq l$, then for almost all $z \in(0,1)$ we have

$$
\left|u_{\alpha}^{(s)}(z)\right| \leq c f(z)^{l-|\alpha|-s-(n-1) / p} U(z),
$$

where

$$
U(z)=\left(\int_{|y|<f(z)}\left|\left(\nabla_{l} u\right)(y, z)\right|^{p} d y\right)^{1 / p} .
$$

Proof. Since $u_{\alpha}(z)=(-f(z))^{-|\alpha|} v_{\alpha}(z)$ with

$$
v_{\alpha}(z)=\int_{|\eta|<1}\left(D^{\alpha} K\right)(\eta) u(f(z) \eta, z) d \eta
$$

and each derivative $v_{\alpha}^{(s)}$ for $s \leq l$ can be obtained by differentiation of the integrand in (3.4), we have $u_{\alpha} \in W_{p}^{l}(\varepsilon, 1-\varepsilon)$ for any small $\varepsilon>0$.

To prove (3.3), first we consider the case where $\alpha=0, s=l$. By (3.2),

$$
(T u)^{(l)}(z)=\int_{|\eta|<1} K(\eta) \frac{\partial^{l}}{\partial z^{l}}[u(f(z) \eta, z)] d \eta .
$$

Using known formulas for high derivatives of composite functions (see Maz'ya [15, Theorem 1.1.7] and Fraenkel [25]), we obtain

$$
\begin{aligned}
\frac{\partial^{l}}{\partial z^{l}}[u(f(z) \eta, z)]= & \left.\frac{\partial^{l} u}{\partial z^{l}}(y, z)\right|_{y=f(z) \eta} \\
& +\sum_{1 \leq|\beta| \leq l}\left(D^{\beta} u\right)(f(z) \eta, z) \eta^{\bar{\beta}} \sum_{\kappa, \lambda} c_{\kappa, \lambda}\left(f^{\left(\kappa_{1}\right)}(z)\right)^{\lambda_{1}} \cdots\left(f^{\left(\kappa_{m}\right)}(z)\right)^{\lambda_{m}},
\end{aligned}
$$

where the summation is taken over all multiindices $\beta=\left(\bar{\beta}, \beta_{n}\right) \in \mathbb{Z}_{+}^{n}$ and $\kappa, \lambda \in \mathbb{Z}_{+}^{m}$ such that $|\bar{\beta}|>0$,

$$
\sum_{i=1}^{m} \lambda_{i}=|\bar{\beta}|, \quad \sum_{i=1}^{m} \lambda_{i} \kappa_{i}=l-\beta_{n} ; \quad \lambda_{i}, \kappa_{i} \geq 1, \quad i=1, \ldots, m,
$$

and the $c_{\kappa, \lambda}$ are numerical coefficients. Since $\left|f^{\left(\kappa_{i}\right)}(z)\right| \leq c f(z)^{1-\kappa_{i}}$, the modulus of the general term in the sum over $\kappa, \lambda$ is at most $c f(z)^{|\beta|-l}$. Thus, we arrive at the following representation:

$$
(T u)^{(l)}(z)=\sum_{1 \leq|\beta| \leq l} \psi_{\beta}(z) I_{\beta}(z), \quad z \in(0,1) .
$$

Here $\beta=\left(\bar{\beta}, \beta_{n}\right) \in \mathbb{Z}_{+}^{n},|\bar{\beta}|>0$ for $|\beta|<l$,

$$
I_{\beta}(z)=\int_{|\eta|<1} K(\eta) \eta^{\bar{\beta}}\left(D^{\beta} u\right)(f(z) \eta, z) d \eta,
$$

and the $\psi_{\beta}$ are measurable functions on $(0,1)$ satisfying

$$
\left|\psi_{\beta}(z)\right| \leq c f(z)^{|\beta|-l}, \quad 1 \leq|\beta| \leq l .
$$


Let $|\beta|<l$, and let $v(\eta, z)=\left(D^{\beta} u\right)(f(z) \eta, z)$. We fix $z \in(0,1)$ and denote by $Q$ an arbitrary polynomial in $\mathcal{P}_{l-|\beta|-1}^{(n-1)}$. Using (3.1), we obtain

$$
\begin{aligned}
\left|I_{\beta}(z)\right| & =\left|\int_{|\eta|<1} K(\eta) \eta^{\bar{\beta}}(v(\eta, z)-Q(\eta)) d \eta\right| \\
& \leq c \inf _{Q}\|v(\cdot, z)-Q\|_{p, B_{1}} .
\end{aligned}
$$

Remark 3.1 shows that the right-hand side of (3.8) does not exceed

$$
c\left\|\left(\nabla_{l-|\beta|}^{\prime} v\right)(\cdot, z)\right\|_{p, B_{1}},
$$

where $\left(\nabla_{l-|\beta|}^{\prime} v\right)(\eta, z)$ is the gradient of order $l-|\beta|$ in $\eta_{1}, \ldots, \eta_{n-1}$. The substitution $\eta=y / f(z)$ gives

$$
\left|I_{\beta}(z)\right| \leq c f(z)^{(1-n) / p+l-|\beta|} U(z)
$$

Estimate (3.9) remains valid for $|\beta|=l$. Here (3.9) follows from (3.6) by Hölder's inequality. Combining (3.5), (3.7), and (3.9) yields

$$
\left|(T u)^{(l)}(z)\right| \leq c f(z)^{(1-n) / p} U(z) .
$$

Thus, (3.3) is established for $s=l$ and $\alpha=0$. By replacing $u$ with $D^{\alpha} u$ and $l$ with $l-|\alpha|$ in (3.10), we also obtain (3.3) for $0 \leq|\alpha|<l$ and $s=l-|\alpha|$.

The case where $l \geq s>l-|\alpha|,|\alpha|>0$. In this case the following representation is deduced by analogy with (3.5):

$$
\begin{aligned}
u_{\alpha}^{(s)}(z)= & \sum_{1 \leq|\beta| \leq l-|\alpha|} \varphi_{\beta}(z) \int_{|\eta|<1} K(\eta) \eta^{\bar{\beta}}\left(D^{\mu} u\right)(f(z) \eta, z) d \eta \\
& +\sum_{i=1}^{s+|\alpha|-l} \sum_{|\gamma|=l-|\alpha|} \sigma_{i, \gamma}(z) \frac{d^{i}}{d z^{i}} \int_{|\eta|<1} K_{\gamma}(\eta)\left(D^{\nu} u\right)(f(z) \eta, z) d \eta
\end{aligned}
$$

Here

$$
\begin{aligned}
& \beta=\left(\bar{\beta}, \beta_{n}\right) \in \mathbb{Z}_{+}^{n}, \quad \mu=\left(\alpha+\bar{\beta}, \beta_{n}\right), \\
& \gamma=\left(\bar{\gamma}, \gamma_{n}\right) \in \mathbb{Z}_{+}^{n}, \quad \nu=\left(\alpha+\bar{\gamma}, \gamma_{n}\right),
\end{aligned}
$$

and the functions $\varphi_{\beta}, \sigma_{i, \gamma}$ are measurable on $(0,1)$ and satisfy

$$
\left|\varphi_{\beta}(z)\right| \leq c f(z)^{|\beta|-s}, \quad\left|\sigma_{i, \gamma}(z)\right| \leq c f(z)^{i+|\gamma|-s} .
$$

Furthermore, $|\bar{\beta}|>0$ if $|\beta|<l-|\alpha|$, and the $K_{\gamma} \in C_{0}^{\infty}\left(B_{1}^{(n-1)}\right)$ are certain standard functions. By (3.1), we have

$$
\int K(\eta) \eta^{\bar{\beta}} Q(\eta) d \eta=0 \quad \text { if }|\beta|<l-|\alpha|, Q \in \mathcal{P}_{l-|\mu|-1}^{(n-1)},
$$

and the modulus of the integral in the sum over $\beta$ in (3.11) is estimated with the help of the generalized Poincaré inequality in the same way as (3.9) was deduced from (3.8). Thus, the modulus of the general term in this sum is majorized by the right-hand side of (3.3).

Now, we turn to the estimation of the double sum in (3.11). Let $I_{\alpha, \gamma}(z)$ denote the last-written integral in (3.11). We have

$$
I_{\alpha, \gamma}(z)=(-f(z))^{-|\alpha|} S_{\alpha, \gamma}(z),
$$


where

$$
S_{\alpha, \gamma}(z)=\int_{|\eta|<1} K_{\alpha, \gamma}(\eta)\left(D^{\gamma} u\right)(f(z) \eta, z) d \eta
$$

and $K_{\alpha, \gamma}(\eta)=\left(D^{\alpha} K_{\gamma}\right)(\eta)$. Therefore,

$$
\left|\frac{d^{i}}{d z^{i}} I_{\alpha, \gamma}(z)\right| \leq c \sum_{j=0}^{i}\left|\frac{d^{i-j}}{d z^{i-j}}\left(f(z)^{-|\alpha|}\right) \frac{d^{j}}{d z^{j}} S_{\alpha, \gamma}(z)\right| .
$$

The modulus of the first factor in the general term of the last sum does not exceed $c f(z)^{j-i-|\alpha|}$. Hence,

$$
\left|\sigma_{i, \gamma}(z) \frac{d^{i}}{d z^{i}} I_{\alpha, \gamma}(z)\right| \leq c \sum_{j=0}^{i} f(z)^{|\gamma|-s-|\alpha|+j}\left|\frac{d^{j}}{d z^{j}} S_{\alpha, \gamma}(z)\right| .
$$

To bound the second factor in the general term, we first consider the case where $j=0$. Since $\int K_{\alpha, \gamma} Q d \eta=0$ for all $Q \in \mathcal{P}_{|\alpha|-1}^{(n-1)}$, it follows that

$$
\left|S_{\alpha, \gamma}(z)\right| \leq \inf _{Q}\|w(\cdot, z)-Q\|_{p, B_{1}^{(n-1)}},
$$

where $w(\eta, z)=\left(D^{\gamma} u\right)(f(z) \eta, z)$. Using Remark 3.1 and then making the substitution $\eta=y / f(z)$, we arrive at

$$
\left|S_{\alpha, \gamma}(z)\right| \leq c f(z)^{|\alpha|+(1-n) / p} U(z) .
$$

Thus, the term on the right in (3.13) that corresponds to $j=0$ is not greater than the right-hand side of (3.3).

Let $1 \leq j \leq i$. Then $j+|\gamma| \leq s \leq l$, and $S_{\alpha, \gamma}^{(j)}(z)$ is obtained by differentiation of the integrand in (3.12), so that

$$
\frac{d^{j}}{d z^{j}} S_{\alpha, \gamma}(z)=\sum_{1 \leq|\delta| \leq j} g_{\delta}(z) J_{\delta}(z)
$$

Here $\delta=\left(\bar{\delta}, \delta_{n}\right) \in \mathbb{Z}_{+}^{n}$,

$$
J_{\delta}(z)=\int_{|\eta|<1} K_{\alpha, \gamma}(\eta) \eta^{\bar{\delta}}\left(D^{\delta+\gamma} u\right)(f(z) \eta, z) d \eta,
$$

and $\left|g_{\delta}(z)\right| \leq c f(z)^{|\delta|-j}$. Note that if $|\delta|<|\alpha|$, then the function

$$
B_{1}^{(n-1)} \ni \eta \mapsto \eta^{\bar{\delta}} K_{\alpha, \gamma}(\eta)
$$

has zero moments up to the order $|\alpha|-|\delta|-1$. Consequently,

$$
\left|J_{\delta}(z)\right| \leq c \inf \left\{\|w(\cdot, z)-Q\|_{p, B_{1}^{(n-1)}}: Q \in \mathcal{P}_{|\alpha|-|\delta|-1}^{(n-1)}\right\}
$$

with $w(\eta, z)=\left(D^{\delta+\gamma} u\right)(f(z) \eta, z)$. Since $w(\cdot, z) \in W_{p}^{|\alpha|-|\delta|}\left(B_{1}^{(n-1)}\right)$ for almost all $z \in$ $(0,1)$, the generalized Poincaré inequality applies. Thus, the above infimum is dominated by

$$
c\left\|\left(\nabla_{|\alpha|-|\delta|}^{\prime} w\right)(\cdot, z)\right\|_{p, B_{1}^{(n-1)}},
$$

where $\nabla_{|\alpha|-|\delta|}^{\prime}$ is the gradient in the first $n-1$ variables. Recalling that $|\alpha|=l-|\gamma|$, we obtain

$$
\left|g_{\delta}(z) J_{\delta}(z)\right| \leq c f(z)^{|\alpha|-j+(1-n) / p} U(z)
$$

If $|\alpha|=|\delta|$, then $|\delta+\gamma|=l$, and $\left|J_{\delta}(z)\right|$ is estimated with the help of Hölder's inequality. Here (3.15) is true again. Estimates (3.13)-(3.15) imply that the modulus of 
the general term of the double sum in (3.11) does not exceed the right-hand side of (3.3). The proof of the lemma is complete.

\section{§4. Proof of Theorems $1.1-1.3$}

For the proof of Theorem 1.1, we need two known facts. One of them is the existence of a $W_{p}^{l}$-preserving extension operator from the peak (1.3) to a circular peak (see [16] and [18, Subsection 5.4.1]).

Lemma 4.1. Let $\Omega \subset \mathbb{R}^{n}$ be a domain of the form (1.3) with $\omega \in C^{0,1}$. If $M \geq 1$ and

$$
G=\left\{x=(y, z) \in \mathbb{R}^{n}:|y|<M \varphi(z), z \in(0,1)\right\},
$$

then for any $1 \leq p \leq \infty$ and any $l=1,2, \ldots$ there exists a continuous linear operator $E: W_{p}^{l}(\Omega) \rightarrow W_{p}^{l}(G)$ that is an extension operator, i.e., $\left.E u\right|_{\Omega}=u$ for all $u \in W_{p}^{l}(\Omega)$.

The second fact we need is Hardy's two-weight inequality for high derivatives on intervals of the real axis. The following result is due to Stepanov 26].

Lemma 4.2. Suppose $-\infty \leq a<b \leq \infty, 1<p \leq q<\infty$, and $l \geq 1$. The existence of a constant $C$ independent of $f$ and such that

$$
\left(\int_{a}^{b}\left|w(x) \int_{x}^{b}(t-x)^{l-1} f(t) d t\right|^{q} d x\right)^{1 / q} \leq C\left(\int_{a}^{b}|v(x) f(x)|^{p} d x\right)^{1 / p}
$$

is equivalent to the fact that the following quantities $A_{0}, A_{1}$ are finite:

$$
\begin{aligned}
A_{\gamma} & =\sup _{z \in(a, b)} A_{\gamma}(z, a, b), \\
A_{\gamma}(z, a, b) & =\left(\int_{a}^{z}(z-t)^{q(l-1)(1-\gamma)}|w(t)|^{q} d t\right)^{\frac{1}{q}}\left(\int_{z}^{b}(t-z)^{\frac{p(l-1) \gamma}{p-1}}|v(t)|^{\frac{p}{1-p}} d t\right)^{\frac{p-1}{p}} .
\end{aligned}
$$

Moreover, if $C$ is the best constant in (4.1), then

$$
\max \left\{A_{0}, A_{1}\right\} \leq C \leq c(p, q, l) \max \left\{A_{0}, A_{1}\right\} .
$$

Next, the operator $L_{p, v}(a, b) \ni f \mapsto g \in L_{q, w}(a, b)$ that acts from the space $L_{p, v}(a, b)$ with the norm $\|f\|_{L_{p, v}(a, b)}=\|f v\|_{L_{p}(a, b)}$ to the space $L_{q, w}(a, b)$ with the norm $\|g\|_{L_{q, w}(a, b)}=$ $\|g w\|_{L_{q}(a, b)}$ by the formula

$$
g(x)=\int_{x}^{b}(t-x)^{l-1} f(t) d t
$$

is compact if and only if $A_{0}$ and $A_{1}$ are finite and

$$
\lim _{z \rightarrow a} A_{\gamma}(z, a, b)=\lim _{z \rightarrow b} A_{\gamma}(z, a, b)=0, \quad \gamma \in\{0 ; 1\} .
$$

For $p=1 \leq q<\infty$, inequality (4.1) is true with the best constant

$$
C=\sup _{z \in(a, b)}|v(z)|^{-1}\left(\int_{a}^{z}(z-t)^{q(l-1)}|w(t)|^{q} d t\right)^{1 / q},
$$

and for $q=\infty, p>1$ the best constant in (4.1) is

$$
C=\sup _{z \in(a, b)}|w(z)|\left(\int_{z}^{b}(t-z)^{(l-1) p /(p-1)}|v(t)|^{-p /(p-1)} d t\right)^{(p-1) / p} .
$$

Below we denote by $c$ various positive constants depending only on $n, p, q, l, \omega$, and $\varphi$. By definition, $a \sim b$ means that $c^{-1} \leq a / b \leq c$. 
Proof of Theorem 1.1. Necessity of (1.5) and the condition $\max \left\{A_{0}, A_{1}\right\}<\infty$. Suppose $W_{p}^{l}(\Omega)$ is continuously imbedded in $L_{q}(\Omega)$. We insert the function

$$
\Omega \ni x=(y, z) \mapsto u(x)=\int_{z}^{1}(t-z)^{l-1} f(t) d t, \quad f \in L_{p, \text { loc }}(0,1),
$$

into the inequality

$$
\|u\|_{L_{q}(\Omega)} \leq c\|u\|_{W_{p}^{l}(\Omega)} .
$$

Then, by Lemma 2.1, (4.2) takes the form

$$
\left(\int_{0}^{1} \varphi(z)^{n-1} d z\left|\int_{z}^{1}(t-z)^{l-1} f(t) d t\right|^{q}\right)^{\frac{1}{q}} \leq c\left(\int_{0}^{1}|f(z)|^{p} \varphi(z)^{n-1} d z\right)^{\frac{1}{p}} .
$$

Now, relation (1.5) for $p=1$ and the finiteness of the quantities $A_{0}, A_{1}$ defined in (1.4) for $p>1$ follow from Lemma 4.2.

Sufficiency. Suppose that $p>1$ and that the quantities (1.4) are finite for $\gamma=0$ and $\gamma=1$. We shall show that (4.2) is fulfilled for all $u \in W_{p}^{l}(\Omega)$.

It is well known (see, e.g., [15, Subsection 1.1.9] and [18, Subsections 1.3.2, 1.3.3]) that the domain $\omega$ in (1.3), having the cone property, is the union of a finite number of domains of class $C^{0,1}$. Since for every such domain there is a class-preserving extension to a circular peak (see Lemma 4.1), there is no loss of generality in assuming that $\Omega$ in (1.3) is a circular peak, i.e., $\omega=B_{1}^{(n-1)}$. Furthermore, Lemma 2.2 allows us to assume also that $\varphi$ in (1.3) has the additional properties

$$
\varphi \in C^{l}((0,1]), \quad\left|\varphi^{(k)}(z)\right| \leq c \varphi(z)^{1-k}, \quad k=1, \ldots, l, \quad z \in(0,1) .
$$

Let $\delta \in(0,1)$ be fixed. Suppose $u \in W_{p}^{l}(\Omega), u(x)=0$ for $z>\delta$. In this case we shall prove that

$$
\|u\|_{L_{q}(\Omega)} \leq c A(\delta)\left\|\nabla_{l} u\right\|_{L_{p}(\Omega)},
$$

where

$$
A_{\gamma}(z, \delta)=\left(\int_{0}^{z}(z-t)^{q(l-1)(1-\gamma)} \varphi(t)^{n-1} d t\right)^{\frac{1}{q}}\left(\int_{z}^{\delta} \varphi(t)^{\frac{n-1}{1-p}}(t-z)^{\frac{p(l-1) \gamma}{p-1}} d t\right)^{\frac{p-1}{p}} .
$$

First, observe that the finiteness of $A_{0}, A_{1}$ implies the boundedness of $A(\delta)$ for $\delta \in(0,1]$, because the quantity (4.6) is dominated by $A_{\gamma}$ (see (1.4)). Below, we shall establish the estimate

$$
A(\delta) \geq c \varphi(\delta)^{l-n / p+n / q},
$$

which, in particular, implies that $l-n / p+n / q \geq 0$ (because the left-hand side of (4.7) is bounded); hence, $q$ does not exceed the limit exponent in Sobolev's imbedding. It suffices to check (4.7) for small $\delta>0$. Using (4.5) and (4.6), we obtain

$$
\begin{aligned}
A(\delta) & \geq A_{1}(\delta-\varphi(\delta), \delta) \\
& \geq\left(\int_{\delta-2 \varphi(\delta)}^{\delta-\varphi(\delta)} \varphi(t)^{n-1} d t\right)^{1 / q}\left(\int_{\delta-\varphi(\delta)}^{\delta} \varphi(t)^{\frac{n-1}{1-p}}(t-\delta+\varphi(\delta))^{(l-1) p^{\prime}} d t\right)^{1 / p^{\prime}} .
\end{aligned}
$$


Since $\varphi(\delta+O(\varphi(\delta)))=\varphi(\delta)+o(\varphi(\delta))$, the right-hand side of the above inequality is not less than

$$
c \varphi(\delta)^{n / q} \varphi(\delta)^{(1-n) / p}\left(\int_{0}^{\varphi(\delta)} t^{(l-1) p^{\prime}} d t\right)^{1 / p^{\prime}}
$$

which dominates the right-hand side of (4.7). Thus, (4.7) is established.

We note that if (4.4) is valid for the functions vanishing for $z>\delta$, then it is also valid for all $u \in W_{p}^{l}(\Omega)$. To see this, we observe that, for $\varepsilon \in(0,1)$, the truncated peak $\Omega^{(\varepsilon)}=\{x \in \Omega: z>\varepsilon\}$ belongs to the class $C^{0,1}$, whence $W_{p}^{l}\left(\Omega^{(\varepsilon)}\right)$ is continuously imbedded in $L_{q}\left(\Omega^{(\varepsilon)}\right)$ by Sobolev's theorem (because $l-n / p+n / q \geq 0$ ).

Let $K \in C_{0}^{\infty}\left(B_{1}^{(n-1)}\right)$, and let (3.1) be fulfilled, along with $\int K(y) d y=1$. For $\alpha \in \mathbb{Z}_{+}^{n-1},|\alpha|<l$, we define

$$
u_{\alpha}(z)=\varphi(z)^{1-n} \int_{|y|<\varphi(z)} K(y / \varphi(z))\left(D_{y}^{\alpha} u\right)(y, z) d y, \quad z \in(0,1),
$$

and consider the "polynomial"

$$
Q(x)=\sum_{|\alpha|<l} u_{\alpha}(z) y^{\alpha} / \alpha !, \quad x=(y, z) \in \Omega .
$$

Our purpose is to prove the estimates

$$
\begin{aligned}
\|Q\|_{L_{q}(\Omega)} & \leq c A(\delta)\left\|\nabla_{l} u\right\|_{L_{p}(\Omega)}, \\
\|u-Q\|_{L_{q}(\Omega)} & \leq c A(\delta)\left\|\nabla_{l} u\right\|_{L_{p}(\Omega)},
\end{aligned}
$$

which imply (4.4) and, thus, the "if" part of the theorem.

Proof of inequality (4.8). Let

$$
Q_{\alpha}(x)=u_{\alpha}(z) y^{\alpha}, \quad \alpha \in \mathbb{Z}_{+}^{n-1},|\alpha|<l .
$$

Since $u_{\alpha}(z)=0$ for $z>\delta$, and there exists $u_{\alpha}^{(l)} \in L_{p \text {, loc }}(0,1)$ (see Lemma 3.1), we have

$$
u_{\alpha}(z)=\frac{(-1)^{l}}{(l-1) !} \int_{z}^{\delta}(t-z)^{l-1} u_{\alpha}^{(l)}(t) d t
$$

for a.e. $z \in(0, \delta)$. Clearly

$$
\left\|Q_{\alpha}\right\|_{L_{q}(\Omega)} \leq c\left(\int_{0}^{\delta} \varphi(z)^{q|\alpha|+n-1}\left|u_{\alpha}(z)\right|^{q} d z\right)^{1 / q} .
$$

To bound the right-hand side of (4.11), we use Lemma 4.2. Identity (4.10) and Lemma 4.2 show that the best constant $C(\delta)$ in the inequality

$$
\left(\int_{0}^{\delta} \varphi(z)^{q|\alpha|+n-1}\left|u_{\alpha}(z)\right|^{q} d z\right)^{1 / q} \leq C(\delta)\left(\int_{0}^{\delta} \varphi(z)^{p|\alpha|+n-1}\left|u_{\alpha}^{(l)}(z)\right|^{p} d z\right)^{1 / p}
$$

satisfies

$$
\begin{aligned}
C(\delta) \sim \max _{\gamma \in\{0 ; 1\}} \sup _{z \in(0, \delta)}\{ & \left(\int_{0}^{z}(z-t)^{\varphi}(t)^{n-1+q|\alpha|} d t\right)^{\frac{1}{q}} \\
& \left.\times\left(\int_{z}^{\delta} \varphi(\tau)^{\frac{n-1+p|\alpha|}{1-p}}(\tau-z)^{p^{\prime}(l-1) \gamma} d \tau\right)^{\frac{1}{p}}\right\}, \quad \frac{1}{p}+\frac{1}{p^{\prime}}=1 .
\end{aligned}
$$


By the monotonicity of $\varphi$, the product of the last two integrals in braces does not exceed the quantity (4.6), so that (4.12) is true with $C(\delta)=c A(\delta)$. By Lemma 3.1, we have

$$
\varphi(z)^{p|\alpha|+n-1}\left|u_{\alpha}^{(l)}(z)\right|^{p} \leq c \int_{|y|<\varphi(z)}\left|\left(\nabla_{l} u\right)(y, z)\right|^{p} d y
$$

for a.e. $z \in(0,1)$. Therefore, the expression on the right in $(4.12)$ is not greater than the right-hand side of (4.8). Thus, for all $\alpha \in \mathbb{Z}_{+}^{n-1}$ with $|\alpha|<l$, the left-hand side of (4.11) is dominated by $c A(\delta)\left\|\nabla_{l} u\right\|_{L_{p}(\Omega)}$. Estimate (4.8) is established.

Proof of inequality (4.9). We define a sequence $\left\{z_{k}\right\}_{k \geq 0}$ by

$$
z_{0}=\delta, \quad z_{k+1}+\varphi\left(z_{k+1}\right)=z_{k}, \quad k \geq 0 .
$$

Then

Consider the "cells"

$$
z_{k} \searrow 0, \quad z_{k+1} z_{k}^{-1} \rightarrow 1, \quad \varphi\left(z_{k+1}\right) \varphi\left(z_{k}\right)^{-1} \rightarrow 1 .
$$

$$
\Omega_{k}=\left\{x: z \in\left(z_{k+1}, z_{k}\right),|y|<\varphi(z)\right\}, \quad k \geq 0 .
$$

Since $\Omega_{k} \in C^{0,1}$ and $l-n / p+n / q \geq 0$, Sobolev's theorem gives

$$
\|v\|_{L_{q}\left(\Omega_{k}\right)} \leq c \varphi\left(z_{k}\right)^{n / q-n / p}\left(\|v\|_{L_{p}\left(\Omega_{k}\right)}+\varphi\left(z_{k}\right)^{l}\left\|\nabla_{l} v\right\|_{L_{p}\left(\Omega_{k}\right)}\right),
$$

where $k=0,1, \ldots$ and $v \in W_{p}^{l}\left(\Omega_{k}\right)$ is an arbitrary function. Note that the map

$$
W_{p}^{l}\left(B_{\varphi(z)}^{(n-1)}\right) \ni u(\cdot, z) \mapsto Q(\cdot, z)
$$

is a projection from $W_{p}^{l}\left(B_{\varphi(z)}^{(n-1)}\right)$ to $\mathcal{P}_{l-1}^{(n-1)}$ (see the example at the beginning of $\S 3$ ). By Remark 3.1, the estimate

$$
\int_{|y|<\varphi(z)}|u(y, z)-Q(y, z)|^{p} d y \leq c \varphi(z)^{l p} \int_{|y|<\varphi(z)}\left|\nabla_{l} u(y, z)\right|^{p} d y
$$

is true for a.e. $z \in(0, \delta)$. Combining this with (4.13) for $v=u-Q$, we see that

$$
\|u-Q\|_{L_{q}\left(\Omega_{k}\right)} \leq c \varphi\left(z_{k}\right)^{l-n / p+n / q}\left(\left\|\nabla_{l} u\right\|_{L_{p}\left(\Omega_{k}\right)}+\left\|\nabla_{l} Q\right\|_{L_{p}\left(\Omega_{k}\right)}\right) .
$$

Now, we estimate $\left\|\nabla_{l} Q\right\|_{L_{p}\left(\Omega_{k}\right)}$. Let

$$
\alpha=\left(\alpha_{1}, \ldots, \alpha_{n-1}\right), \quad \bar{\gamma}=\left(\gamma_{1}, \ldots, \gamma_{n-1}\right), \quad \gamma=\left(\bar{\gamma}, \gamma_{n}\right)
$$

be multiindices such that $|\alpha|<l,|\gamma|=l$. We put $Q_{\alpha}(x)=u_{\alpha}(z) y^{\alpha}$, as before. If $\gamma_{i}>\alpha_{i}$ for some $i=1, \ldots, n-1$, then $D^{\gamma} Q_{\alpha}(x)=0$. Suppose $\bar{\gamma} \leq \alpha$ (i.e., $\gamma_{i} \leq \alpha_{i}$ for all $i=1, \ldots, n-1)$. In this case

$$
\left|D^{\gamma} Q_{\alpha}(x)\right| \leq c \varphi(z)^{|\alpha|-|\bar{\gamma}|}\left|u_{\alpha}^{\left(\gamma_{n}\right)}(z)\right|, \quad x=(y, z) \in \Omega .
$$

By Lemma 3.1, we have

$$
\left|D^{\gamma} Q_{\alpha}(x)\right|^{p} \leq c \varphi(z)^{1-n} \int_{|y|<\varphi(z)}\left|\nabla_{l} u(y, z)\right|^{p} d y,
$$

whence

$$
\left\|\nabla_{l} Q_{\alpha}\right\|_{L_{p}\left(\Omega_{k}\right)} \leq c\left\|\nabla_{l} u\right\|_{L_{p}\left(\Omega_{k}\right)}, \quad k \geq 0 .
$$

This estimate and (4.14) yield

$$
\|u-Q\|_{L_{q}\left(\Omega_{k}\right)} \leq c \varphi\left(z_{k}\right)^{l-n / p+n / q}\left\|\nabla_{l} u\right\|_{L_{p}\left(\Omega_{k}\right)}, \quad k \geq 0 .
$$

Using the algebraic inequality

$$
\left(\sum_{k} a_{k}^{q}\right)^{1 / q} \leq\left(\sum_{k} a_{k}^{p}\right)^{1 / p}, \quad a_{k} \geq 0, \quad q \geq p
$$


from (4.15) we deduce that

$$
\|u-Q\|_{L_{q}(\Omega)} \leq c \varphi(\delta)^{l-n / p+n / q}\left\|\nabla_{l} u\right\|_{L_{p}(\Omega)} .
$$

Recalling (4.7), we arrive at (4.9). The theorem is established for $p>1$.

With obvious modifications, the above proof covers the case of $p=1$ as well. Under condition (1.5), similar (and even somewhat simpler) arguments lead to (4.4) with $p=1$, $u \in W_{1}^{l}(\Omega), u(x)=0$ for $z>\delta$, and

$$
A(\delta)=\sup _{z \in(0, \delta)} \varphi(z)^{1-n}\left(\int_{0}^{z}(z-t)^{(l-1) q} \varphi(t)^{n-1} d t\right)^{1 / q} .
$$

The proof of Theorem 1.1 is complete.

Proof of Theorem 1.2. Sufficiency of conditions (1.6), (1.7). The argument mentioned at the beginning of the proof of the sufficiency part in Theorem 1.1 enables us to restrict ourselves to the case where $\omega=B_{1}^{(n-1)}$ in (1.3). Also, we may assume that $\varphi$ satisfies (4.3). Under these conditions, in Theorem 1.1 we obtained estimate (4.4), where $\delta \in(0,1)$ is any fixed number, $u \in W_{p}^{l}(\Omega)$ is an arbitrary function with $u(y, z)=0$ for $z>\delta$, and $A(\delta)$ is defined in (4.5)-(4.6) for $p>1$ and in (4.16) for $p=1$. Suppose

$$
\eta \in C^{\infty}([0, \infty)), \quad 0 \leq \eta \leq 1,\left.\quad \eta\right|_{[0,1 / 2]}=1,\left.\quad \eta\right|_{(1, \infty)}=0 .
$$

If $\delta \in(0,1 / 2)$, we put $\eta_{\delta}(x)=\eta(z / \delta)$ for $x=(y, z) \in \Omega$. By the aforementioned, for $u \in W_{p}^{l}(\Omega)$ we have

$$
\|u\|_{L_{q}(\Omega)} \leq c A(\delta)\left\|\nabla_{l}\left(\eta_{\delta} u\right)\right\|_{L_{p}(\Omega)}+\|u\|_{L_{q}\left(\Omega^{(\delta / 2)}\right)},
$$

where

It follows that

$$
\Omega^{(\varepsilon)}=\left\{x=(y, z) \in \mathbb{R}^{n}: z \in(\varepsilon, 1),|y|<\varphi(z)\right\} .
$$

$$
\|u\|_{q, \Omega} \leq c A(\delta)\left\|\nabla_{l} u\right\|_{L_{p}(\Omega)}+M_{\delta}\|u\|_{W_{p}^{l-1}\left(\Omega^{(\delta / 2)}\right)}+\|u\|_{L_{q}\left(\Omega^{(\delta / 2)}\right)}
$$

with a positive constant $M_{\delta}$ independent of $u$. We observe that the assumptions of the theorem imply $\lim _{\delta \rightarrow 0} A(\delta)=0$, and estimate (4.7) implies $l-n / p+n / q>0$. Therefore, the space $W_{p}^{l}\left(\Omega^{(\varepsilon)}\right)$ is compactly imbedded in $L_{q}\left(\Omega^{(\varepsilon)}\right)$ and in $W_{p}^{l-1}\left(\Omega^{(\varepsilon)}\right)$ for any $\varepsilon \in(0,1)$ (see [2, §11] and [3]). Now estimate (4.17) allows us to apply the well-known diagonal method to show that any sequence bounded in $W_{p}^{l}(\Omega)$ admits a subsequence convergent in $L_{q}(\Omega)$.

Necessity of conditions (1.6), (1.7). Let $W_{p}^{l}(\Omega)$ be compactly imbedded in $L_{q}(\Omega)$ for $p>1$. We denote by $U$ the unit ball in the space of functions $f \in L_{p, \text { loc }}(0,1)$ with finite norm $\left\|f \varphi^{(n-1) / p}\right\|_{L_{p}(0,1)}$. Then the set of functions

$$
\Omega \ni(y, z) \mapsto(T f)(y, z)=\int_{z}^{1}(t-z)^{l-1} f(t) d t
$$

with $f \in U$ is bounded in $W_{p}^{l}(\Omega)$ by Lemma 2.1. Thus, there exists a sequence $\left\{f_{k}\right\}_{k \geq 1} \subset$ $U$ such that $\left\{T f_{k}\right\}$ is convergent in $L_{q}(\Omega)$, which is equivalent to the convergence of the sequence

$$
(0,1) \ni z \mapsto \int_{z}^{1}(t-z)^{l-1} f_{k}(t) d t, \quad k=1,2, \ldots,
$$

in the space of functions $g \in L_{q, \text { loc }}(0,1)$ with finite norm $\left\|g \varphi^{(n-1) / q}\right\|_{L_{q}(0,1)}$. So, if we view $T f$ in (4.18) as a function of $z \in(0,1)$, then the operator $f \mapsto T f$, viewed as acting in the above weighted function spaces on the interval $(0,1)$, is compact. Hence, (1.6) follows by Lemma 4.2. 
Suppose $W_{1}^{l}(\Omega)$ is compactly imbedded in $L_{q}(\Omega)$. If (1.7) fails, then there are two sequences $\left\{r_{k}\right\},\left\{\varepsilon_{k}\right\}$ of positive numbers such that $\lim r_{k}=\lim \varepsilon_{k}=0$ and

$$
\varphi\left(r_{k}+\varepsilon_{k}\right)^{1-n}\left(\int_{0}^{r_{k}}\left(r_{k}-t\right)^{(l-1) q} \varphi(t)^{n-1} d t\right)^{1 / q} \geq \mathrm{const}>0 .
$$

We define

$$
u_{k}(x)=\int_{z}^{1}(t-z)^{l-1} \chi_{\left(r_{k}, r_{k}+\varepsilon_{k}\right)}(t) d t, \quad x=(y, z) \in \Omega
$$

(here $\chi_{E}$ is the characteristic function of $E$ ). Then, by Lemma 2.1,

$$
\left\|u_{k}\right\|_{W_{1}^{l}(\Omega)} \sim\left\|\nabla_{l} u_{k}\right\|_{L_{1}(\Omega)} \leq c \int_{r_{k}}^{r_{k}+\varepsilon_{k}} \varphi(z)^{n-1} d z \leq c \varphi\left(r_{k}+\varepsilon_{k}\right)^{n-1} \varepsilon_{k} .
$$

The definition of $u_{k}$ implies that

$$
\begin{aligned}
\left\|u_{k}\right\|_{L_{q}(\Omega)}^{q} & \geq c \int_{0}^{r_{k}} \varphi(z)^{n-1} d z\left(\int_{r_{k}}^{r_{k}+\varepsilon_{k}}(t-z)^{l-1} d t\right)^{q} \\
& \geq c \varepsilon_{k}^{q} \int_{0}^{r_{k}} \varphi(z)^{n-1}\left(r_{k}-z\right)^{(l-1) q} d z .
\end{aligned}
$$

Thus, for the sequence $v_{k}=u_{k} /\left\|u_{k}\right\|_{W_{1}^{l}(\Omega)}$ we have

$$
\left\|v_{k}\right\|_{L_{q}(\Omega)} \geq \frac{c}{\varphi\left(r_{k}+\varepsilon_{k}\right)^{n-1}}\left(\int_{0}^{r_{k}}\left(r_{k}-t\right)^{(l-1) q} \varphi(t)^{n-1} d t\right)^{\frac{1}{q}} \geq \text { const }>0 .
$$

By the compactness of the imbedding operator $W_{1}^{l}(\Omega) \rightarrow L_{q}(\Omega)$, some subsequence of $\left\{v_{k}\right\}$ converges in $L_{q}(\Omega)$. The preceding inequality says that this limit in $L_{q}(\Omega)$ is a nonzero function. But since $v_{k}(x)=0$ for $z>r_{k}+\varepsilon_{k}$, if a subsequence of $\left\{v_{k}\right\}$ converges in $L_{q}(\Omega)$, then its limit equals zero almost everywhere on $\Omega$. This contradiction proves (1.7), completing the proof of the theorem.

Proof of Corollary 1.1. Let $p^{\prime}=p /(p-1)$. Using (4.6) and the monotonicity of $\varphi$, we deduce the inequality

$$
\begin{aligned}
A_{\gamma}(z, \delta) & \leq z^{1 / q+(l-1)(1-\gamma)} \varphi(z)^{\frac{n-1}{q}}\left(\int_{z}^{\delta} \varphi(t)^{\frac{n-1}{1-p}} t^{(l-1) p^{\prime} \gamma} d t\right)^{1 / p^{\prime}} \\
& \leq z^{1 / q}\left(\int_{z}^{\delta} t^{(l-1) p^{\prime}} \varphi(t)^{(n-1)(1 / q-1 / p) p^{\prime}} d t\right)^{1 / p^{\prime}} \\
& \leq z^{1 / q} \Psi(\delta)\left(\int_{z}^{\infty} t^{-p^{\prime} / q} \frac{d t}{t}\right)^{1 / p^{\prime}} \leq\left(q / p^{\prime}\right)^{1 / p^{\prime}} \Psi(\delta)
\end{aligned}
$$

where $\Psi(\delta)=\sup \{\Phi(t): t \in(0, \delta)\}$. Thus, $A_{\gamma}$ in (1.4) does not exceed the supremum in (1.8) multiplied by a positive constant depending only on $p, q$.

If $p=1$, we have

$$
\varphi(z)^{1-n}\left(\int_{0}^{z}(z-t)^{(l-1) q} \varphi(t)^{n-1} d t\right)^{1 / q} \leq \Phi(z), \quad z \in(0,1) .
$$

So, (1.8) implies (1.5) for $p=1$ and the finiteness of $A_{0}, A_{1}$ for $p>1$.

Let (1.9) be true. Then (1.7) is also true by (4.20). We check (1.6) for $p>1$. Using (4.19), for $0<z<\delta<1$ we obtain

$$
A_{\gamma}(z) \leq c(p, q) \sup _{t \in(0, \delta)} \Phi(t)+\left(\int_{0}^{z} g(z, t) d t\right)^{1 / q}\left(\int_{\delta}^{1} h(z, t) d t\right)^{1 / p^{\prime}},
$$


where

$$
g(z, t)=(z-t)^{(l-1) q(1-\gamma)} \varphi(t)^{n-1}, \quad h(z, t)=(t-z)^{(l-1) p^{\prime} \gamma} \varphi(t)^{\frac{1-n}{p-1}} .
$$

Let $\varepsilon>0$. By $(1.9)$, there exists a number $\delta \in(0,1)$ such that the first summand on the right in (4.21) is less than $\varepsilon / 2$. To bound the second summand, which is the product of two factors, we observe that the first factor tends to zero as $z \rightarrow 0$, while the second factor is majorized by $\varphi(\delta)^{(1-n) / p}$. Hence, for fixed $\delta \in(0,1)$, there exists a number $\zeta \in(0, \delta)$ such that the product of the factors is less than $\varepsilon / 2$ for all $z \in(0, \zeta)$. For the same $z$ we have $A_{\gamma}(z)<\varepsilon$. Relations (1.6) are established.

Now, the sufficiency of (1.8) and (1.9) for the continuity or compactness of the imbedding operator $W_{p}^{l}(\Omega) \rightarrow L_{q}(\Omega)$ is a consequence of Theorems 1.1 and 1.2.

Assume that $\varphi(2 z) \sim \varphi(z), z \in(0,1 / 2)$. By the definition of $A_{\gamma}(z)$ (see Theorem 1.1), for $p>1$ we have

$$
\begin{aligned}
A_{1}(z) & \geq\left(\int_{z / 2}^{z} \varphi(t)^{n-1} d t\right)^{1 / q}\left(\int_{z}^{2 z} \varphi(t)^{\frac{1-n}{p-1}}(t-z)^{(l-1) p^{\prime}} d t\right)^{1 / p^{\prime}} \\
& \geq c\left(z \varphi(z)^{n-1}\right)^{1 / q} \varphi(z)^{(1-n) / p}\left(\int_{0}^{z} \tau^{(l-1) p^{\prime}} d \tau\right)^{1 / p^{\prime}}
\end{aligned}
$$

whence $A_{1}(z) \geq c \Phi(z)$.

It is also easily checked that for $p=1$ the left-hand side of (4.20) does not exceed $c \Phi(z)$. Now, the necessity of conditions (1.8), (1.9) follows from Theorems 1.1, 1.2. The proof of Corollary 1.1 is finished.

Proof of Theorem 1.3. Necessity. Let $W_{p}^{l}(\Omega)$ be continuously imbedded in $C(\Omega) \cap L_{\infty}(\Omega)$. To verify (1.10), we define

$$
u_{\delta}(x)=\int_{z}^{1}(t-z)^{l-1} g_{\delta}(t) d t, \quad x=(y, z) \in \Omega,
$$

where $\delta$ is a small positive parameter,

$$
g_{\delta}(t)=t^{(l-1) /(p-1)} \varphi(t)^{(1-n) /(p-1)} \chi_{(\delta, 1-\delta)}(t),
$$

and $\chi_{E}$ designates the characteristic function of $E$. Then $u_{\delta}(x)=0$ for $z>1-\delta$, $u_{\delta} \in C^{l-1}(\Omega) \cap W_{\infty}^{l}(\Omega)$, and furthermore, $\left|\left(\nabla_{l} u\right)(x)\right| \sim g_{\delta}(z)$ for $x \in \Omega$. Since

$$
\left\|u_{\delta}\right\|_{W_{p}^{l}(\Omega)} \leq c\left\|\nabla_{l} u_{\delta}\right\|_{L_{p}(\Omega)}
$$

by Lemma 2.1, we have

$$
\left\|u_{\delta}\right\|_{W_{p}^{l}(\Omega)}^{p} \leq c I_{\delta}, \quad I_{\delta}=\int_{\delta}^{1-\delta} t^{(l-1) p /(p-1)} \varphi(t)^{(1-n) /(p-1)} d t .
$$

Let $x=(y, z) \in \Omega, z \in(0, \delta)$. For such an $x$ we have

$$
u_{\delta}(x)=\int_{\delta}^{1}(t-z)^{l-1} g_{\delta}(t) d t \leq c\left\|u_{\delta}\right\|_{W_{p}^{l}(\Omega)} .
$$

By the dominated convergence theorem, in the above integral we can pass to the limit as $z \rightarrow 0$, so that

$$
I_{\delta}=\int_{\delta}^{1} t^{l-1} g_{\delta}(t) d t \leq c I_{\delta}^{1 / p}
$$

Since $I_{\delta}<\infty$, it follows that

$$
\int_{\delta}^{1-\delta} t^{(l-1) p /(p-1)} \varphi(t)^{(1-n) /(p-1)} d t \leq c .
$$


But $\delta>0$ is arbitrarily small; therefore, inequality (1.10) is a consequence of the lastwritten estimate and Fatou's lemma.

Suppose $W_{1}^{l}(\Omega)$ is continuously imbedded in $C(\Omega) \cap L_{\infty}(\Omega)$. If (1.11) fails, then there exists a sequence $\left\{r_{k}\right\}$ of positive numbers such that $r_{k} \rightarrow 0$ and $r_{k}^{l-1} \varphi\left(r_{k}\right)^{1-n} \rightarrow \infty$. Let $\eta$ be the function introduced in the proof of sufficiency in Theorem 1.2. We define

$$
u_{k}(x)=r_{k}^{l-1} \varphi\left(r_{k}\right)^{1-n} \eta\left(z / r_{k}\right), \quad x=(y, z) \in \Omega, k=1,2, \ldots .
$$

Then $u_{k} \in C^{\infty}(\Omega), u_{k}(x)=0$ for $z>r_{k}$, and $u_{k}(x)=r_{k}^{l-1} \varphi\left(r_{k}\right)^{1-n}$ for $z \in\left(0, r_{k} / 2\right)$. Furthermore,

$$
\left\|\nabla_{l} u_{k}\right\|_{L_{1}(\Omega)} \leq c r_{k}^{-1} \varphi\left(r_{k}\right)^{1-n} \int_{r_{k} / 2}^{r_{k}} \varphi(z)^{n-1} d z \leq c .
$$

This and Lemma 2.1 show that $\left\{u_{k}\right\}$ is a bounded sequence in $W_{1}^{l}(\Omega)$. However, we have

$$
\left\|u_{k}\right\|_{L_{\infty}(\Omega)} \geq r_{k}^{l-1} \varphi\left(r_{k}\right)^{1-n} \rightarrow \infty
$$

which contradicts the continuity of the imbedding $W_{1}^{l}(\Omega) \subset L_{\infty}(\Omega)$. Thus, $(1.11)$ is true.

Suppose $W_{1}^{l}(\Omega)$ is compactly imbedded in $C(\Omega) \cap L_{\infty}(\Omega)$. If (1.12) fails, then there is a positive infinitesimal sequence $\left\{r_{k}\right\}$ for which

$$
\inf \left\{r_{k}^{l-1} \varphi\left(r_{k}\right)^{1-n}\right\}=c>0 \text {. }
$$

We may assume without loss of generality that $r_{k+1}<r_{k} / 2$ for $k=1,2, \ldots$ As has been shown above, the sequence $\left\{u_{k}\right\}$ constructed in (4.22) is bounded in $W_{1}^{l}(\Omega)$. Suppose that $i>k, z \in\left(r_{i}, r_{k} / 2\right)$, and $x=(y, z) \in \Omega$. For such an $x$ we have $u_{i}(x)=0$, $u_{k}(x)=r_{k}^{l-1} \varphi\left(r_{k}\right)^{1-n}$, whence $\left\|u_{i}-u_{k}\right\|_{L_{\infty}(\Omega)} \geq c$, and there is no subsequence of $\left\{u_{k}\right\}$ convergent in $L_{\infty}(\Omega)$. So, we have established (1.12) by contradiction; this concludes the proof of the necessity part.

Sufficiency. Assuming (1.10), we show that the imbedding $W_{p}^{l}(\Omega) \subset C(\Omega) \cap L_{\infty}(\Omega)$ is compact. As in the sufficiency proofs in the preceding theorems, we can restrict ourselves to the case where $\omega=B_{1}^{(n-1)}$ in (1.3). Also, we may assume that $\varphi$ satisfies (4.3).

Suppose $\delta \in(0,1), u \in W_{p}^{l}(\Omega)$, and $u(y, z)=0$ for $z>\delta$. We shall prove (4.4) with $q=\infty$ and

$$
A(\delta)=\left(\int_{0}^{\delta} \frac{z^{(l-1) p^{\prime}}}{\varphi(z)^{(n-1) /(p-1)}} d z\right)^{1 / p^{\prime}}, \quad 1 / p+1 / p^{\prime}=1
$$

Since

$$
A(\delta) \geq c \varphi(\delta)^{(1-n) / p} \delta^{l-1+1 / p^{\prime}} \geq c \varphi(\delta)^{l-n / p}
$$

and $A(\delta) \rightarrow 0$ as $\delta \rightarrow 0$, it follows that $l p>n$. In particular, Sobolev's theorem says that any $u \in W_{p}^{l}(\Omega)$ agrees with a function of class $C(\Omega)$ almost everywhere.

As in Theorem 1.1, we introduce a quasipolynomial $Q$ and check estimates (4.8) and (4.9) for $q=\infty$. The proof of (4.9) repeats the corresponding arguments in Theorem 1.1, leading to (4.15) for $q=\infty$. By (4.15), we have

$$
\|u-Q\|_{L_{\infty}(\Omega)} \leq c \varphi(\delta)^{l-n / p}\left\|\nabla_{l} u\right\|_{L_{p}(\Omega)} .
$$

Combining this with (4.24), we arrive at (4.9).

We turn to (4.8). As in Theorem 1.1, we define $Q_{\alpha}(x)=u_{\alpha}(z) y^{\alpha}$ for $\alpha \in \mathbb{Z}_{+}^{n-1}$, $|\alpha|<l$. Then

$$
\left\|Q_{\alpha}\right\|_{L_{\infty}(\Omega)} \leq\left\|u_{\alpha} \varphi^{|\alpha|}\right\|_{L_{\infty}(0,1)}
$$


Using (4.10) and Lemma 4.2, we see that the best constant $C(\delta)$ in the inequality

$$
\operatorname{ess}_{\sup _{z \in(0,1)}}\left\{\varphi(z)^{|\alpha|}\left|u_{\alpha}(z)\right|\right\} \leq C(\delta)\left(\int_{0}^{\delta} \varphi(z)^{p|\alpha|+n-1}\left|u_{\alpha}^{(l)}(z)\right|^{p} d z\right)^{1 / p}
$$

is given by

$$
C(\delta)=\sup _{z \in(0,1)} \varphi(z)^{|\alpha|}\left(\int_{z}^{\delta}(t-z)^{(l-1) p^{\prime}} \varphi(t)^{(n-1+p|\alpha|) /(1-p)} d t\right)^{1 / p^{\prime}} .
$$

Since $C(\delta)$ is dominated by the quantity (4.23), it follows that $\left\|Q_{\alpha}\right\|_{L_{\infty}(\Omega)}$ does not exceed the right-hand side of $(4.25)$ with $C(\delta)$ replaced by $A(\delta)$. It remains to observe that this right-hand side is majorized by $c A(\delta)\left\|\nabla_{l} u\right\|_{L_{p}(\Omega)}$ by Lemma 3.1. This yields (4.8).

Let $u \in W_{p}^{l}(\Omega)$ be an arbitrary function. As in the proof of Theorem 1.2, we introduce a smooth cut-off function $\eta_{\delta}(x)=\eta(z / \delta)$ and obtain the estimate

$$
\|u\|_{L_{\infty}(\Omega)} \leq c A(\delta)\left\|\nabla_{l} u\right\|_{L_{p}(\Omega)}+M(\delta)\|u\|_{W_{p}^{l-1}\left(\Omega^{(\delta / 2)}\right)}+\|u\|_{L_{\infty}\left(\Omega^{(\delta / 2)}\right)},
$$

where $\Omega^{(\varepsilon)}=\{x \in \Omega: z>\varepsilon\}$, and $M(\delta)>0$ is independent of $u$. Since $l p>n$ and $\Omega^{(\varepsilon)} \in C^{0,1}$ for $\varepsilon \in(0,1)$, the space $W_{p}^{l}\left(\Omega^{(\varepsilon)}\right)$ is compactly imbedded in $L_{\infty}\left(\Omega^{(\varepsilon)}\right)$ and in $W_{p}^{l-1}\left(\Omega^{(\varepsilon)}\right)$ (see [2, $\left.\delta 11\right]$, [3] ). Furthermore, we have $\lim _{\delta \rightarrow 0} A(\delta)=0$. Now the relative compactness in $L_{\infty}(\Omega)$ of a set bounded in $W_{p}^{l}(\Omega)$ can be deduced from (4.26) by a standard argument. The proof of sufficiency for $p>1$ is concluded. With obvious modifications, this proof also extends to the case where $p=1$.

Suppose that the domain $\omega$ in (1.3) belongs to the class $C^{0,1}$. By Lemma 4.1, any function in $W_{p}^{l}(\Omega)$ has a class-preserving extension to the circular peak $G=\{(y, z)$ : $z \in(0,1),|y|<\varphi(z)\}$. The boundary of $G$ can be represented locally by the graph of a continuous function; therefore (see $\left[3\right.$ and [15, Subsection 1.1.6]), the set $C^{\infty}(\bar{G})$ is dense in $W_{p}^{l}(G)$. Then, clearly, $C^{\infty}(\bar{\Omega})$ is dense in $W_{p}^{l}(\Omega)$. Thus, under the assumptions of Theorem 1.3, every function in $W_{p}^{l}(\Omega)$ is the limit in $L_{\infty}(\Omega)$ of a sequence in $C(\bar{\Omega})$ and, hence, agrees with a function of class $C(\bar{\Omega})$ almost everywhere. The proof of Theorem 1.3 is complete.

\section{Application of Theorem 1.1 to the Neumann problem}

Let $\Omega$ be a bounded domain in $\mathbb{R}^{n}, l$ a positive integer, and $a_{\alpha \beta} \in L_{\infty}(\Omega)$, where $\alpha, \beta$ are $n$-dimensional multiindices satisfying $|\alpha|=|\beta|=l$. Suppose that $a_{\alpha \beta}=a_{\beta \alpha}$ and there exists a constant $c>0$ such that

$$
\sum_{|\alpha|=|\beta|=l} a_{\alpha \beta}(x) \xi_{\alpha} \xi_{\beta} \geq c \sum_{|\alpha|=l} \xi_{\alpha}^{2}
$$

for all vectors $\left\{\xi_{\alpha}\right\}_{|\alpha|=l}$ with real components. Furthermore, let $a \in L_{\infty}(\Omega), a(x) \geq$ const $>0$.

For $2 \leq q<\infty$, the operator $A_{q}$ of the Neumann problem for the differential operator

$$
u \mapsto(-1)^{l} \sum_{|\alpha|=|\beta|=l} D^{\alpha}\left(a_{\alpha \beta} D^{\beta} u\right)+a u
$$

is defined by the following conditions:

1) $u \in L_{q}(\Omega) \cap L_{2}^{l}(\Omega), A_{q} u \in L_{q^{\prime}}(\Omega), 1 / q+1 / q^{\prime}=1$; 
2) for all $v \in L_{q}(\Omega) \cap L_{2}^{l}(\Omega)$,

$$
\int_{\Omega} v A_{q} u d x=\int_{\Omega}\left(\sum_{|\alpha|=|\beta|=l} a_{\alpha \beta}(x) D^{\beta} u D^{\alpha} v+a(x) u v\right) d x .
$$

It is known (see [15, 4.10] and [27]) that if the set $L_{q}(\Omega) \cap L_{2}^{l}(\Omega)$ is dense in $W_{2}^{l}(\Omega)$, then the solvability of the equation

$$
A_{q} u=f
$$

for all $f \in L_{q^{\prime}}(\Omega)$ is equivalent to the continuity of the imbedding $W_{2}^{l}(\Omega) \subset L_{q}(\Omega)$.

Applying this assertion to the domain (1.3) and using Theorem 1.1, we arrive at the following statement.

Let $\Omega$ be a peak in $\mathbb{R}^{n}$ of the form (1.3) with $\omega \in C^{0,1}$. Then the Neumann problem (4.27) is uniquely solvable for all $f \in L_{q^{\prime}}(\Omega), q \geq 2$, if and only if

$$
\sup _{z \in(0,1)}\left\{\left(\int_{0}^{z}(z-t)^{q(l-1)} \varphi(t)^{n-1} d t\right)^{1 / q}\left(\int_{z}^{1} \varphi(t)^{1-n} d t\right)^{1 / 2}\right\}<\infty
$$

and

$$
\sup _{z \in(0,1)}\left\{\left(\int_{0}^{z} \varphi(t)^{n-1} d t\right)^{1 / q}\left(\int_{z}^{1} \varphi(t)^{1-n}(t-z)^{2(l-1)} d t\right)^{1 / 2}\right\}<\infty .
$$

\section{$\S 5$. HÖLDER DOMAINS}

Here we use the above results to state conditions for the continuity of the imbeddings $W_{p}^{l}(\Omega) \subset L_{q}(\Omega)$ and $W_{p}^{l}(\Omega) \subset C(\bar{\Omega})$ on the so-called Hölder domains. First, we describe this class of domains.

Let $a \in(0, \infty]$, and let $\psi:[0, a) \rightarrow[0, \infty)$ be a concave monotone increasing function such that $\psi(0)=0$. We say that a bounded domain $\Omega \subset \mathbb{R}^{n}(n \geq 2)$ belongs to the class $C^{0, \psi} 1$ if for every point $X \in \partial \Omega$ there exists a local Cartesian system $x=\left(x^{\prime}, x_{n}\right): x^{\prime} \in$ $\mathbb{R}^{n-1}, x_{n} \in \mathbb{R}^{1}$, with origin $X$ and a cylindrical neighborhood $U=\left\{\left(x^{\prime}, x_{n}\right):\left|x^{\prime}\right|<r\right.$, $\left.x_{n} \in(-b, b)\right\}, r<a / 2$, such that

$$
U \cap \Omega=\left\{\left(x^{\prime}, x_{n}\right):\left|x^{\prime}\right|<r,-b<x_{n}<f\left(x^{\prime}\right)\right\}
$$

and

$$
U \cap \partial \Omega=\left\{\left(x^{\prime}, x_{n}\right):\left|x^{\prime}\right|<r, x_{n}=f\left(x^{\prime}\right)\right\},
$$

where $f$ is a function satisfying

$$
f\left(x^{\prime}\right) \in(-b, b), \quad\left|f\left(x^{\prime}\right)-f\left(y^{\prime}\right)\right| \leq \psi\left(\left|x^{\prime}-y^{\prime}\right|\right), \quad x^{\prime}, y^{\prime} \in \bar{B}_{r}^{(n-1)} .
$$

We observe that if $x=\left(x^{\prime}, x_{n}\right) \in U \cap \bar{\Omega}, y=\left(y^{\prime}, y_{n}\right) \in U$, and

$$
x_{n}-y_{n}>\psi\left(\left|x^{\prime}-y^{\prime}\right|\right),
$$

then $y_{n}<f\left(y^{\prime}\right)$, i.e., $y \in U \cap \Omega$. Hence, if $\Omega \in C^{0, \psi}$, then there exists a number $\varepsilon>0$ such that every point in $\bar{\Omega}$ is the vertex of a peak contained in $\Omega$ and congruent to the peak

$$
\left\{x: x_{n} \in(0, \varepsilon),\left|x^{\prime}\right|<\psi^{-1}\left(x_{n}\right)\right\} .
$$

Let $\varphi=\psi^{-1}$. Then $\varphi$ is a continuous, monotone increasing, and convex function on $[0, \varepsilon]$. Therefore, $\varphi$ satisfies the Lipschitz condition on the same interval, and the limit

\footnotetext{
${ }^{1}$ If $\psi(t)=t^{\lambda}, \lambda \in(0,1]$, this class is denoted by $C^{0, \lambda}$.
} 
$\lim _{t \rightarrow+0} \varphi^{\prime}(t) \geq 0$ exists. If this limit is positive, then $\Omega$ has the cone property, and thus, Sobolev's theorem is valid. In what follows we assume that

$$
\varphi(0)=\lim _{t \rightarrow+0} \varphi^{\prime}(t)=0 .
$$

Theorem 5.1. Let $\Omega \subset \mathbb{R}^{n}$ be a domain of class $C^{0, \psi}$, and let $\varphi=\psi^{-1}$.

(i) If $l$ is a positive integer, $1 \leq p \leq q<\infty$, and

$$
\sup \left\{t^{l-1 / p+1 / q} \varphi(t)^{(n-1)(1 / q-1 / p)}: t \in[0, \varepsilon]\right\}<\infty
$$

for some $\varepsilon>0$, then the space $W_{p}^{l}(\Omega)$ is continuously imbedded in $L_{q}(\Omega)$.

(ii) Suppose that

$$
\int_{0}^{\varepsilon} t^{(l-1) p^{\prime}} \varphi(t)^{(1-n) p^{\prime} / p} d t<\infty, \quad 1 / p+1 / p^{\prime}=1,
$$

for some $\varepsilon>0$. Then $W_{p}^{l}(\Omega)$ is continuously imbedded in $C(\bar{\Omega})$.

For the proof, we need the well-known "transitivity property" of the imbeddings $L_{p}^{l}(\Omega) \subset L_{q}(\Omega)$ (see, e.g., [15, 4.9]).

Lemma 5.1. Let $\Omega \subset \mathbb{R}^{n}$ be a domain of finite volume. If the space $L_{1}^{1}(\Omega)$ is continuously imbedded in $L_{s}(\Omega)$ for $s \geq 1$, then, for any positive integer $l$ and any $p \in[1, \infty)$ satisfying $p l\left(1-s^{-1}\right)<1$, the space $L_{p}^{l}(\Omega)$ is continuously imbedded in $L_{q}(\Omega)$ with

$$
q=p /\left(1-p l\left(1-s^{-1}\right)\right) .
$$

$L_{p}^{l}(\Omega)$ is continuously imbedded in $L_{q}(\Omega)$ for any $q<\infty$ if $p l\left(1-s^{-1}\right)=1$.

Proof of Theorem 5.1. (i) First, we note that the spaces $W_{p}^{l}(\Omega)$ and $L_{p}^{l}(\Omega)$ coincide if $\Omega \in C^{0, \psi}$ (see [15, Subsection 1.1.11]).

Let $[0, \varepsilon] \ni t \mapsto t^{1 / s} \varphi(t)^{(1-n)(1-1 / s)}$ be a bounded function for some $\varepsilon>0, s \geq 1$. Then, in accordance with a result by Labutin (see $\left[21\right.$ ), the space $W_{1}^{1}(\Omega)$ is continuously imbedded in $L_{s}(\Omega)$.

Suppose (5.2) is fulfilled; we define $s$ by equation (5.4). Then $s \geq 1$ and the left-hand side of (5.2) equals

$$
\left(\sup \left\{t^{1 / s} \varphi(t)^{(1-n)(1-1 / s)}: t \in[0, \varepsilon]\right\}\right)^{l} .
$$

By Lemma 5.1 and the aforementioned, $W_{p}^{l}(\Omega)$ is continuously imbedded in $L_{q}(\Omega)$.

(ii) Let $x \in \bar{\Omega}$, and let $G_{x}$ be a peak with vertex $x$ contained in $\Omega$ and congruent to the peak (5.1). By Theorem 1.3, for all $u \in W_{p}^{l}(\Omega) \cap C(\bar{\Omega})$ the estimate

$$
|u(x)| \leq c\|u\|_{W_{p}^{l}\left(G_{x}\right)}
$$

is valid with a constant independent of $x, u$. Then, clearly,

$$
|u(x)| \leq c\|u\|_{W_{p}^{l}(\Omega)} .
$$

Since the set $W_{p}^{l}(\Omega) \cap C(\bar{\Omega})$ is dense in $W_{p}^{l}(\Omega)$ (see [3]), the continuity of the imbedding $W_{p}^{l}(\Omega) \subset C(\bar{\Omega})$ follows. The proof of the theorem is complete.

Remark 5.1. Condition (5.2) is sharp in the following sense: there is a domain $\Omega \in C^{0, \psi}$ for which (5.2) is necessary for the continuity of the imbedding operator $W_{p}^{l}(\Omega) \rightarrow L_{q}(\Omega)$. Theorem 1.1 and Corollary 1.1 show that the peak (5.1) can play the role of such a domain if $\psi^{-1}(2 z) \leq$ const $\cdot \psi^{-1}(z)$ for small $z>0$.

Condition (5.3) is sharp in the same sense. 


\section{REFERENCES}

[1] S. L. Sobolev, On a theorem of functional analysis, Mat. Sb. 4 (1938), no. 3, 471-497. (Russian)

[2] _ Some applications of functional analysis in mathematical physics, Leningrad. Univ., Leningrad, 1950; English transl. from 3rd Russian ed., Transl. Math. Monogr., vol. 90, Amer. Math. Soc., Providence, RI, 1991. MR0052039 (14:565a) MR.1125990 (92e:46067)

[3] E. Gagliardo, Proprietà di alcune classi di funzioni in più variabili, Ric. Mat. 7 (1958), 102-137. MR0102740 (21:1526)

[4] O. V. Besov, Integral representations of functions and embedding theorems for a domain with a flexible horn condition, Trudy Mat. Inst. Steklov. 170 (1984), 12-30; English transl. in Proc. Steklov Inst. Math. 1987, no. 1 (170). MR790325 (87a:46047)

[5] _ Sobolev's embedding theorem for a domain with an irregular boundary, Mat. Sb. 192 (2001), no. 3, 3-26; English transl., Sb. Math. 192 (2001), no. 3-4, 323-346. MR.1836304 (2002e:46032)

[6] Yu. G. Reshetnyak, Integral representations of differentiable functions in domains with a nonsmooth boundary, Sibirsk. Mat. Zh. 21 (1980), no. 6, 108-116; English transl., Siberian Math. J. 21 (1980), no. 6, 833-839 (1981). MR601195 (83h:26019)

[7] B. Bojarski, Remarks on Sobolev imbedding inequalities, Complex Analysis (Joensuu, 1987), Lecture Notes in Math., vol. 1351, Springer, Berlin, 1988, pp. 52-68. MR.982072 (90b:46068)

[8] S. Buckley and P. Koskela, Sobolev-Poincaré implies John, Math. Res. Lett. 2 (1995), 577-593. MR 1359964 (96i:46035)

[9] P. Hajłasz and P. Koskela, Isoperimetric inequalities and imbedding theorems in irregular domains, J. London Math. Soc. (2) 58 (1998), 425-450. MR1668136 (99m:46079)

[10] T. Kilpeläinen and J. Malý, Sobolev inequalities on sets with irregular boundaries, Z. Anal. Anwendungen 19 (2000), no. 2, 369-380. MR.1768998 (2001g:46075)

[11] S. V. Poborchii, Some counterexamples to the embedding theorems for Sobolev spaces, Vestnik S.Peterburg. Univ. Ser. 1 1998, vyp. 4, 49-58; English transl., Vestnik St. Petersburg Univ. Math. 31 (1998), no. 4, 46-55 (2000). MR1794641 (2001g:46082)

[12] V. G. Maz'ya, Classes of domains and imbedding theorems for function spaces, Dokl. Akad. Nauk SSSR 133 (1960), no. 3, 527-530; English transl., Soviet Math. Dokl. 1 (1960), 882-885. MR0126152 (23:A3448)

[13] , The continuity and boundedness of functions in S. L. Sobolev spaces, Integral and Differential Operators, Differential Equations, Probl. Mat. Anal., No. 4, Leningrad. Univ., Leningrad, 1973, pp. 46-77; English transl., J. Soviet Math. 6 (1976), no. 1, 29-50. MR0348481 (50:979)

[14] _ The summability of functions belonging to Sobolev spaces, Linear and Nonlinear Differential Equations, Differential Operators, Probl. Mat. Anal., No. 5, Leningrad. Univ., Leningrad, 1975, pp. 66-98; English transl. in J. Soviet Math. 10 (1978), no. 6. MR0511931 (58:23545)

[15] _ Sobolev spaces, Leningrad. Univ., Leningrad, 1985; English transl., Springer-Verlag, Berlin, 1985. MR817985 (87g:46056) MR0817985 (87g:46056)

[16] V. G. Maz'ya and S. V. Poborchili, Extension of functions in S. L. Sobolev classes to the exterior of a domain with the vertex of a peak on the boundary. II, Czechoslovak Math. J. 37 (1987), no. 1, 128-150. (Russian) MR875135 (88f:46076b)

[17] _ _ Imbedding theorems for Sobolev spaces in domains with cusps, Linköping Univ., 1992, 34 pp. (Preprint/ LiTH-MAT-R-92-14).

[18] _ Differentiable functions on bad domains, World Sci. Publishing Co., Inc., River Edge, NJ, 1997. MR1643072 (99k:46057)

[19] I. G. Globenko, Some questions in the theory of imbedding for domains with singularities on the boundary, Mat. Sb. (N.S.) 57 (1962), no. 2, 201-224. (Russian) MR0143022 (26:589)

[20] D. A. Labutin, Integral representation of functions and the embedding of Sobolev spaces on domains with zero angles, Mat. Zametki 61 (1997), no. 2, 201-219; English transl., Math. Notes 61 (1997), no. 1-2, 164-179. MR.1619998 (99b:46043)

[21] _ Embedding of Sobolev spaces on Hölder domains, Trudy Mat. Inst. Steklov. 227 (1999), 170-179; English transl., Proc. Steklov Inst. Math. 1999, no. 4 (227), 163-172. MR.1784315 (2001i:46050)

[22] - The unimprovability of Sobolev inequalities for a class of irregular domains, Trudy Mat. Inst. Steklov. 232 (2001), 218-222; English transl., Proc. Steklov Inst. Math. 2001, no. 1 (232), 211-215. MR.1851450 (2002c:46070)

[23] R. A. Adams, Sobolev spaces, Pure Appl. Math., vol. 65, Acad. Press, New York-London, 1975. MR0450957 (56:9247)

[24] M. Fukushima and M. Tomisaki, Construction and decomposition of reflecting diffusions on Lipschitz domains with Hölder cusps, Probab. Theory Related Fields 106 (1996), 521-557. MR1421991 (98d:60157) 
[25] L. E. Fraenkel, Formulae for high derivatives of composite functions, Math. Proc. Cambridge Philos. Soc. 83 (1978), 159-165. MR0486377 (58:6124)

[26] V. D. Stepanov, Two-weight estimates for Riemann-Liouville integrals, Izv. Akad. Nauk SSSR Ser. Mat. 54 (1990), no. 3, 645-656; English transl., Math. USSR-Izv. 36 (1991), no. 3, 669-681. MR $1072699(92 \mathrm{~b}: 44006)$

[27] S. V. Poborchii, On solvability of the Neumann problem for elliptic equations of high order, Vestnik S.-Peterburg. Univ. Ser. 1 1998, vyp. 3, 63-66; English transl., Vestnik St. Petersburg Univ. Math. 31 (1998), no. 3, 57-60 (1999). MR1794685 (2001h:35042)

Department of Mathematics, 58183 Linköping University, Sweden

E-mail address: vlmaz@mai.liu.se

Department of Mathematics and Mechanics, St. Petersburg State University, UniverSitetskiI Prospect 28, Stary ̌ Peterhof, St. Petersburg 198504, Russia

E-mail address: Sergei.Poborchi@paloma.spbu.ru

Received 5/SEP/2005

Translated by S. V. POBORCHI 